\title{
Finite element dynamical subgrid-scale model for low Mach-number flows with radiative heat transfer
}

\author{
Matias Avila, Ramon Codina, Javier Principe \\ Universitat Politècnica de Catalunya \\ Jordi Girona 1-3, Edifici C1. 08034 Barcelona
}

\section{Contents}

1 Introduction $\quad 2$

2 The low Mach number problem coupled with radiative heat transfer 3

2.1 Initial and Boundary Value Problem . . . . . . . . . . . . . . . . . . . 3

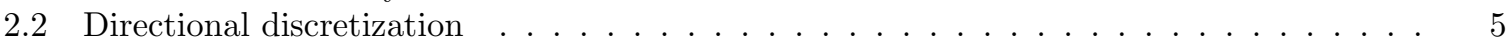

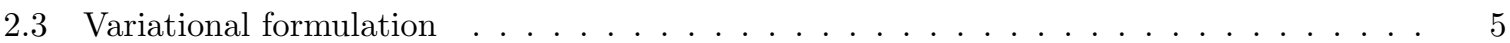

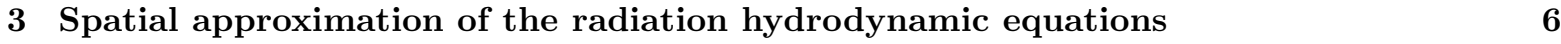

4 Global energy conservation $\quad 9$

5 Linearization strategy of coupling terms between radiation and temperature equations

$6 \quad$ An application example: fire in a 3D room with an open door $\quad 11$

7 Conclusions $\quad 15$ 


\begin{abstract}
Purpose - To present a finite element approximation of the low Mach number equations coupled with radiative equations to account for radiative heat transfer. For high temperature flows this coupling can have strong effects on the temperature and velocity fields.

Design/methodology/approach - The basic numerical formulation has been proposed in previous works. It is based on the variational multiscale concept in which the unknowns of the problem are divided into resolved and subgrid parts which are modeled to consider their effect into the former. The aim of the present article is to extend this modeling to the case in which the low Mach number equations are coupled with radiation, also introducing the concept of subgrid scales for the radiation equations.

Findings - As in the non-radiative case, an important improvement in the accuracy of the numerical scheme is observed when the nonlinear effects of the subgrid scales are taken into account. Besides it is possible to show global conservation of thermal energy.

Originality/value - The original contribution of the work is the proposal of keeping the variational multiscale splitting into the nonlinear coupling between the low Mach number and the radiative transport equations, its numerical evaluation and the description of its properties.

Keywords Radiative heat transfer, Thermal flows, Stabilization, Variational multiscale, Finite element analysis

Paper type Research paper

\section{Introduction}

1 Introduction
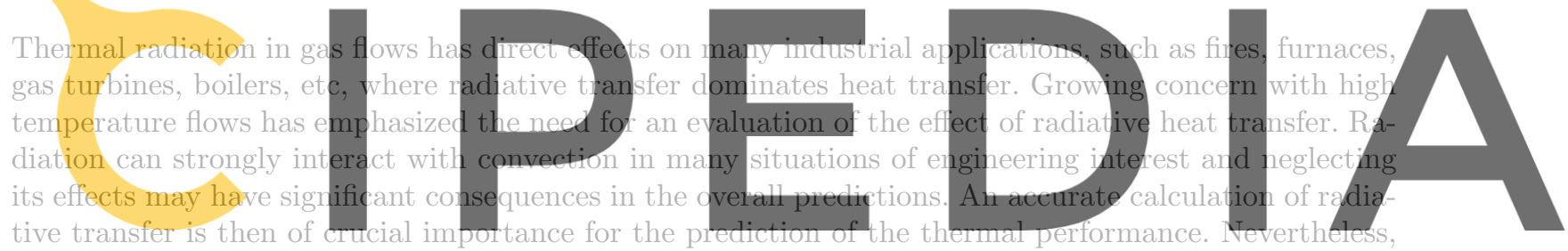

it is common for studies on convective flows to neglect thermal radiation, mainly because the modeling

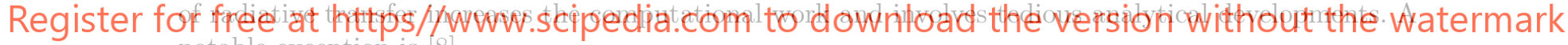
notable exception is [8].

Approximate models for radiative heat transfer have been derived and widely used in the literature.
\end{abstract}

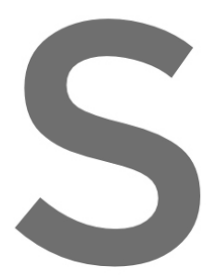

Examples of such approximation are the $P_{N}$ method $[7,13]$ and the discrete ordinates method (DOM) $[15,13]$. Our interest in this work is the development of a nonlinear stabilization technique capable of solving low Mach number flows in radiating media without being focused in a specific radiative model.

Turbulence is the most common state of a fluid in a wide range of technologies and natural conditions. The interaction between turbulence and radiation (TRI) has been demonstrated experimentally, theoretically and numerically, and results from the highly nonlinear coupling between fluctuations of radiation intensity and fluctuations of temperature of the medium [6]. Experimental data and numerical calculations demonstrate that turbulent fluctuations may significantly increase the radiation intensity in both non-luminous and luminous flames. The net radiative power and the fraction of radiative heat loss increase due to TRI. Usually, in combustion applications using large eddy simulation (LES), these interactions are either discarded altogether, or included in the computation without considering any subgrid scale model for radiation.

The focus of the present paper is on how to compute the coupling of the radiative terms in the energy equation and the thermal terms in the radiative equations. In this work we extend the stabilized finite element approximation presented in [3], valid for low Mach number flows, for the presence of radiative heat transfer. The stabilization method is based on the variational multiscale (VMS) method [9], in which a decomposition of the approximating space into a coarse scale resolvable part and a fine scale subgrid part is performed. The distinctive features of our particular approach, discussed in [3], are to consider the subscales as transient and to keep the scale splitting in all the nonlinear terms. The first ingredient permits to obtain better stability and no restrictions on the time step size. The second ingredient permits to prove global conservation properties, gives higher accuracy to the method, and allows us to approach the problem of dealing with thermal turbulence from a strictly numerical point of view, as it was shown in [2]. The aim of the present work is to show that the nonlinear stabilization 
terms coming from the highly nonlinear coupling between temperature and radiation $\left(\kappa \sigma_{B} T^{4}\right)$ are able to model TRI subgrid effects.

The article is organized as follows. In Section 2, the low Mach number equations with radiative coupling and their variational formulation are given. Some different coupling mechanisms between radiative and conductive heat transfer are presented. Afterwards the VMS formulation with dynamic scale splitting is derived in Section 3. Section 4 is devoted to show that this formulation provides global energy conservation when using equal interpolation spaces for pressure and temperature, if the radiation model is globally conservative. The treatment of the coupling of the nonlinear terms is described in detail in Section 5. The formulation is tested in Section 6 and conclusions are drawn in Section 7.

\section{The low Mach number problem coupled with radiative heat} transfer

\subsection{Initial and Boundary Value Problem}

The low Mach number flow equations and the finite element approximation we propose for them were introduced in [3]. When radiative heat transfer is considered the energy equation is modified by adding the divergence of the total radiative heat flux term, $\nabla \cdot q_{r}$. The initial and boundary value problem reads as follows. Let $\Omega \subset \mathbb{R}^{d}$, with $d=2,3$, be the computational domain in which the flow takes place during the time interval $\left[0, t_{\text {end }}\right]$, and let $\partial \Omega$ be its boundary. Let $\mathcal{S}^{2}$ be the unit sphere in $\mathbb{R}^{3}$. The initial and

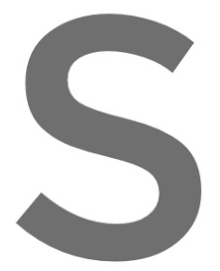
boundary value problem
field $p$, a temperature
that
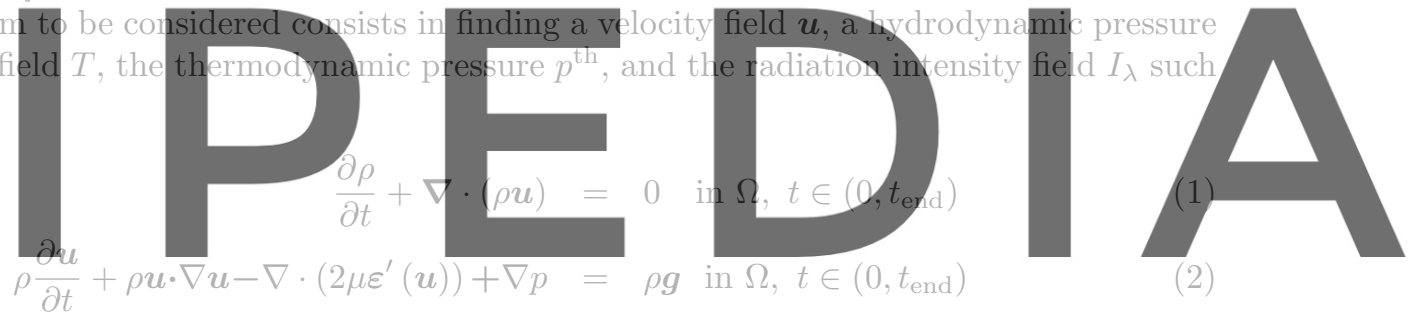

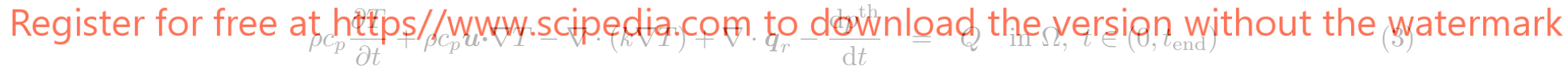

$$
s \cdot \nabla I_{\lambda}+\left(\kappa_{\lambda}+\sigma_{s \lambda}\right) I_{\lambda}-\frac{\sigma_{s \lambda}}{4 \pi} \int_{\mathcal{S}^{2}} \mathrm{~d} s^{\prime} \phi_{\lambda}\left(s^{\prime}, s\right) I_{\lambda}\left(s^{\prime}\right)=\kappa_{\lambda} I_{b \lambda} \quad \text { in } \Omega \times \mathcal{S}^{2}, t \in\left(0, t_{\text {end }}\right)
$$

where $\rho$ denotes the density, $\mu$ the viscosity, $\varepsilon^{\prime}(\boldsymbol{u})=\boldsymbol{\varepsilon}(\boldsymbol{u})-\frac{1}{3}(\boldsymbol{\nabla} \cdot \boldsymbol{u}) \boldsymbol{I}$ the deviatoric part of the rate of deformation tensor $\varepsilon(\boldsymbol{u})=\nabla^{s} \boldsymbol{u}=\frac{1}{2}\left(\boldsymbol{\nabla} \boldsymbol{u}+\boldsymbol{\nabla} \boldsymbol{u}^{T}\right), \boldsymbol{I}$ the identity tensor, $\boldsymbol{g}$ the gravity force vector, $c_{p}$ the specific heat coefficient at constant pressure, $k$ the thermal conductivity and $Q$ the heat source. Equations (1)-(3) represent the mass, momentum and energy conservation, respectively. Equation (4) is the monochromatic radiative transfer equation, and $I_{\lambda}(\boldsymbol{s})$ is the spectral radiative intensity at wavelength $\lambda$ propagating in direction $s$. The function $I_{b \lambda}$ is the spectral blackbody radiation at wavelength $\lambda$, depending only on the temperature $T$. The coefficients $\kappa_{\lambda}$ and $\sigma_{s \lambda}$ are respectively the spectral absorption and scattering coefficients, $\phi_{\lambda}\left(s^{\prime}, s\right)$ is the scattering phase function and $\mathrm{d} s^{\prime}$ the differential of solid angle. The inverse of the light-speed has been considered negligible when writing Eq. (4). Additionally, the state equation for ideal gases is used to give a closure to the system:

$$
\rho=p^{\text {th }} / R T
$$

The radiative heat transfer term in the energy equation, which is the one coupling both problems (also through the boundary conditions, see below), can be expressed in terms of radiative intensity and temperature as

$$
\nabla \cdot \boldsymbol{q}_{r}=\int_{0}^{\infty} \mathrm{d} \lambda \kappa_{\lambda}\left(4 \pi I_{b \lambda}-G_{\lambda}\right)=4 \kappa_{e} \sigma_{B} T^{4}-\kappa_{a} G\left(I_{\lambda}\right)
$$


where $G_{\lambda}$ and $G$ are the spectral and total incident radiation, defined as

$$
\begin{gathered}
G_{\lambda}=\int_{\mathcal{S}^{2}} \mathrm{~d} s I_{\lambda} \\
G\left(I_{\lambda}\right)=\int_{0}^{\infty} \mathrm{d} \lambda G_{\lambda} .
\end{gathered}
$$

The absorption and emission coefficients $\kappa_{a}$ and $\kappa_{e}$ in Eq. (6) are spectral averages of the absorption coefficient $\kappa_{\lambda}$, weighted respectively with the radiation field $G_{\lambda}$ and the blackbody radiation field $I_{b \lambda}$

$$
\begin{aligned}
\kappa_{a} & =\frac{\int_{0}^{\infty} \mathrm{d} \lambda \kappa_{\lambda} G_{\lambda}}{G} \\
\kappa_{e} & =\frac{\pi \int_{0}^{\infty} \mathrm{d} \lambda \kappa_{\lambda} I_{b \lambda}}{\sigma_{B} T^{4}}
\end{aligned}
$$

where $\sigma_{B}$ is the Stephan Boltzmann constant. For a given composition and pressure, the emissivity coefficient only depends on temperature.

The mass, momentum and energy equations must be supplied with initial and boundary conditions. Initial conditions are
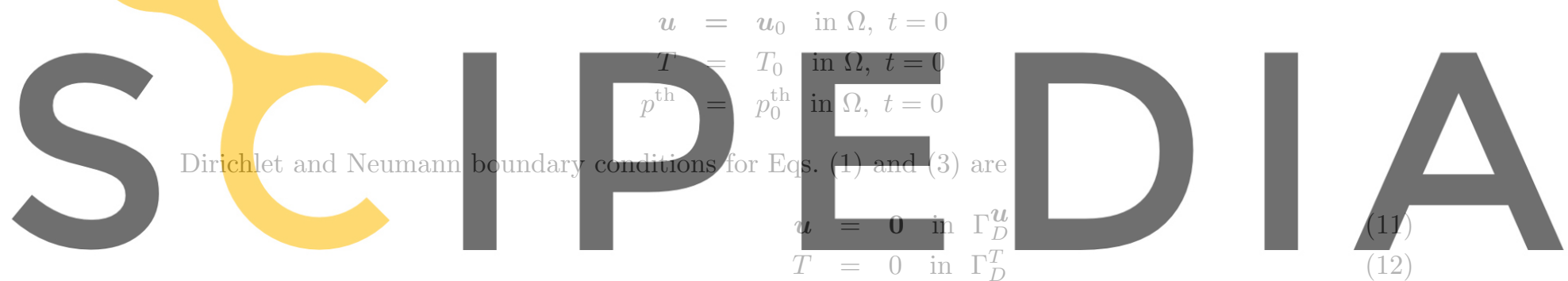

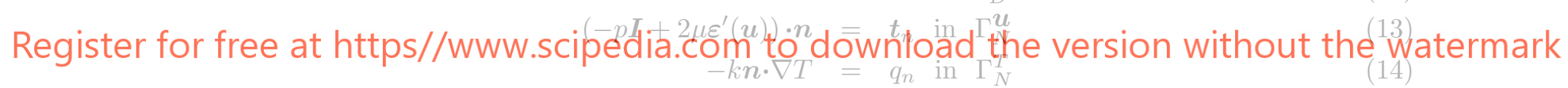

where $\boldsymbol{n}$ is the outer unit normal on the boundary. It is assumed that $\Gamma_{D}^{x} \cup \Gamma_{N}^{x}=\partial \Omega$, and $\Gamma_{D}^{x} \cap \Gamma_{N}^{x}=\emptyset$ for $x=T, \boldsymbol{u}$.

Due to the hyperbolic nature of the radiation equation (4), the intensity entering into the domain $\Omega$ needs to be imposed. The boundary $\Gamma=\partial \Omega \times \mathcal{S}^{2}$ of $\Omega \times \mathcal{S}^{2}$ is divided into the inflow $\Gamma^{-}$and outflow $\Gamma^{+}$boundaries, defined as

$$
\Gamma^{-}=\{(\boldsymbol{x}, \boldsymbol{s}) \in \Gamma \mid \boldsymbol{s} \cdot \boldsymbol{n}<0\}, \quad \Gamma^{+}=\{(\boldsymbol{x}, \boldsymbol{s}) \in \Gamma \mid \boldsymbol{s} \cdot \boldsymbol{n} \geq 0\},
$$

where $\boldsymbol{n}$ is the unit normal vector pointing outwards $\partial \Omega$ at $\boldsymbol{x}$. We shall also make use of the inflow and outflow hemispheres

$$
\mathcal{S}_{\boldsymbol{x}}^{-}:=\left\{\boldsymbol{s} \in \mathcal{S}^{2} \mid \boldsymbol{s} \cdot \boldsymbol{n}<0\right\}, \quad \mathcal{S}_{\boldsymbol{x}}^{+}:=\left\{\boldsymbol{s} \in \mathcal{S}^{2} \mid \boldsymbol{s} \cdot \boldsymbol{n} \geq 0\right\},
$$

which are defined for each $\boldsymbol{x} \in \partial \Omega$.

The radiative transfer equation (4) is subject to emissive and reflective boundary conditions of the form

$$
\left.I_{\lambda}(\boldsymbol{x}, \boldsymbol{s})\right|_{\mathcal{S}_{\boldsymbol{x}}^{-}}=\epsilon_{\lambda} I_{b \lambda}+\frac{r_{\lambda}}{\pi} \int_{\mathcal{S}_{\boldsymbol{x}}^{+}} I_{\lambda}(\boldsymbol{x}, \boldsymbol{s}) \boldsymbol{n} \cdot \boldsymbol{s} \mathrm{d} s
$$

where $\epsilon_{\lambda}$ and $r_{\lambda}$ are respectively the emission and reflective wall coefficients at wavelength $\lambda$. In the present work we consider opaque surfaces, where $r_{\lambda}=1-\epsilon_{\lambda}$.

In some applications the Neumann boundary condition defined by Eq. (14) is changed to impose the complete heat transfer (conductive and radiative) $\mathcal{H}$ through the boundary as

$$
-k \boldsymbol{n} \cdot \nabla T+\boldsymbol{q}_{r} \cdot \boldsymbol{n}=\mathcal{H}
$$


An example is an isolated boundary where the net flux through the boundary must be null $(\mathcal{H}=0)$. This isolated wall is an emmisive and reflective wall, i.e. the radiation boundary condition (15) is satisfied, which integrated over $\mathcal{S}_{\boldsymbol{x}}^{-}$and $\lambda$ permits to obtain

$$
-k \boldsymbol{n} \cdot \nabla T-\epsilon \sigma_{B} T^{4}=\mathcal{H}-(1-r) H
$$

where $H(\boldsymbol{x})$ is the surface irradiation

$$
H(\boldsymbol{x})=\int_{\lambda} d \lambda \int_{\mathcal{S}_{\boldsymbol{x}}^{+}} d s I_{\lambda}(\boldsymbol{x}, \boldsymbol{s}) \boldsymbol{s} \cdot \boldsymbol{n}
$$

and $r$ is the spectral average of $r_{\lambda}$ weighted with the irradiation and $\epsilon$ is the spectral average of $\epsilon_{\lambda}$ weighted with the blackbody radiation $I_{b \lambda}$. This boundary condition is of nonlinear Robin type for the temperature, coupling radiation and temperature in the boundary.

The coupling between the radiation and the hydrodynamic problems is stronger on the boundaries than inside the media when the medium is optically thin. This occurs when the characteristic length of the problem $\left(L_{0}\right)$ is much smaller than the optical length scale $\left(\kappa_{0}^{-1}\right)$, and it is characterized by a small optical length number $\tau=\kappa_{0} L_{0} \ll 1$. On the other hand, when the medium is optically thick it is usual to take $L_{0}=1 / \kappa_{e}$ (i.e. $\tau=1$ ) and in this case the coupling between both problems occurs locally inside the domain. In this case the relative importance of radiative heat and conductive heat transfer is estimated by the Planck number, $\mathrm{Pl}=\frac{k \kappa_{e}}{\sigma_{B} T_{0}^{3}}$, also known as the radiation to conduction parameter [14].
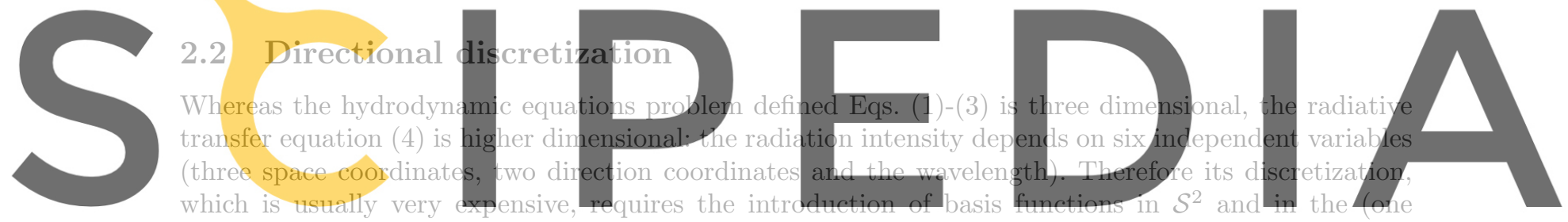

dimensional) wavenumber space. There exist several models to obtain an approximate solution to the

integro-differential equation (4) into a set of coupled differential equations, with an arbitrary number of equations depending on the discretization level. Examples of this kind of models are the discrete ordinates method (DOM), the method of spherical harmonics ( $P_{N}$-approximation), and the ray tracing method. The resulting equations can then be discretized in space using the finite element method, as for example in [11].

The aim of the present paper is to develop a stabilization method for the mass, momentum and energy equations, independent of the radiative model used. We therefore assume that a general directional discretization model is applied to the radiative transfer equation, obtaining directional components of radiation intensity $I_{\lambda}^{d}$. The radiation equation of the adopted model will be denoted as $\mathcal{R}_{M}\left(I_{\lambda}^{d}, T\right)=0$, where $\mathcal{R}_{M}$ is a partial differential operator and $I_{\lambda}^{d}$ the semidiscrete radiation intensity. In the energy equation, the emission coefficient $\kappa_{e}$ and the product of the absorption coefficient and the incident radiation $\kappa_{a} G$ will depend on the obtained radiation intensity distribution $I_{\lambda}^{d}$, and must be given by the radiative model.

\subsection{Variational formulation}

To obtain a variational formulation for the system (1)-(3) together with the radiation model equation, let us denote by $\boldsymbol{V}, Q, W, Z$ the functional spaces where the solution is sought. The corresponding space of test functions will be denoted by $\boldsymbol{V}_{0}, Q_{0}, W_{0}, Z_{0}$

The weak form of the problem consists in finding $\left(\boldsymbol{u}, p, T, I_{\lambda}^{d}\right) \in(\boldsymbol{V}, Q, W, Z)$ such that 


$$
\begin{aligned}
\left(\frac{\partial \rho}{\partial t}+\nabla \cdot(\rho \boldsymbol{u}), q\right)=0 & \forall q \in Q_{0} \\
\left(\rho \frac{\partial \boldsymbol{u}}{\partial t}+\rho \boldsymbol{u} \cdot \nabla \boldsymbol{u}, \boldsymbol{v}\right)+\left(2 \mu \boldsymbol{\varepsilon}^{\prime}(\boldsymbol{u}), \boldsymbol{\varepsilon}^{\prime}(\boldsymbol{v})\right) & \\
-(p, \nabla \cdot \boldsymbol{v})=(\rho \boldsymbol{g}, \boldsymbol{v})+\left(\boldsymbol{t}_{n}, \boldsymbol{v}\right)_{\Gamma_{N}^{\boldsymbol{u}}} & \forall \boldsymbol{v} \in \boldsymbol{V}_{0} \\
\left(\rho c_{p} \frac{\partial T}{\partial t}+\rho c_{p} \boldsymbol{u} \cdot \nabla T+\kappa_{e} \sigma_{B} T^{4}, w\right)+(k \nabla T, \nabla w) & \\
=\left(Q+\frac{\mathrm{d} p^{\mathrm{th}}}{\mathrm{d} t}+\kappa_{a} G\left(I_{\lambda}\right), w\right)+\left(q_{n}, w\right)_{\Gamma_{N}^{T}} & \forall w \in W_{0} \\
\left(\mathcal{R}_{M}\left(I_{\lambda}^{d}, T\right), z\right)=0 & \forall z \in Z_{0}
\end{aligned}
$$

\section{Spatial approximation of the radiation hydrodynamic equa- tions}

Let us consider a finite element partition $\{K\}$ with $n_{e}$ elements of the computational domain $\Omega$, from

which we can construct finite element spaces for the velocity, pressure, temperature and radiation

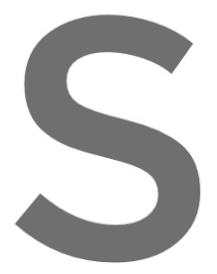

intensity in the usual

respectively.

Let us split the cor

radiation intensity belo

be in principle any spa

split as
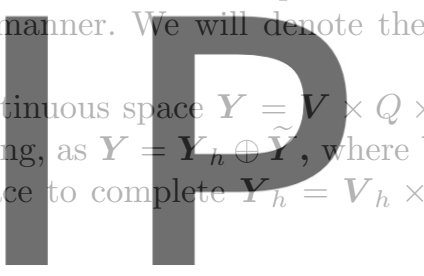

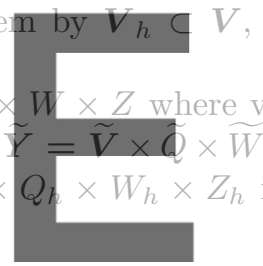

$u=u_{h}+\widetilde{u}$

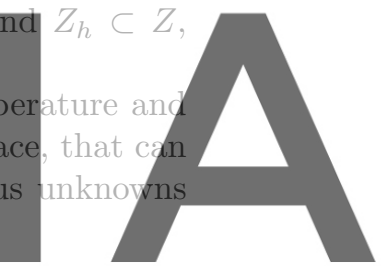

(23)

Register for free at https//www.scipedia.com

$$
\begin{aligned}
T & =T_{h}+\widetilde{T} \\
I_{\lambda h}^{d} & =I_{\lambda h}^{d}+\widetilde{I_{\lambda}^{d}}
\end{aligned}
$$

where the components with subscripts $h$ belong to the corresponding finite element spaces, and the components with the $\sim$ correspond to the subgrid space. These additional components are what we will call subscales.

The spatial approximation will be obtained following the same procedures and approximations done in [3] for the low Mach number equations. The particular approximation consists in keeping time dependency of the subscales and to keep the previous decompositions (23)-(25) in all the terms of the variational problem (19)-(22) even if the differential operator is approximated. It is assumed that the subscales vanish on the interelement boundaries, $\partial K$.

Substituting decompositions (23)-(25) in the variational problem (19)-(21), taking the tests functions in the corresponding finite element spaces and integrating some terms by parts, it is found that the solution $\left(\boldsymbol{u}_{h}, T_{h}, p_{h}, I_{\lambda h}^{d}\right) \in \boldsymbol{V}_{h} \times Q_{h} \times W_{h} \times Z_{h}$ must satisfy 


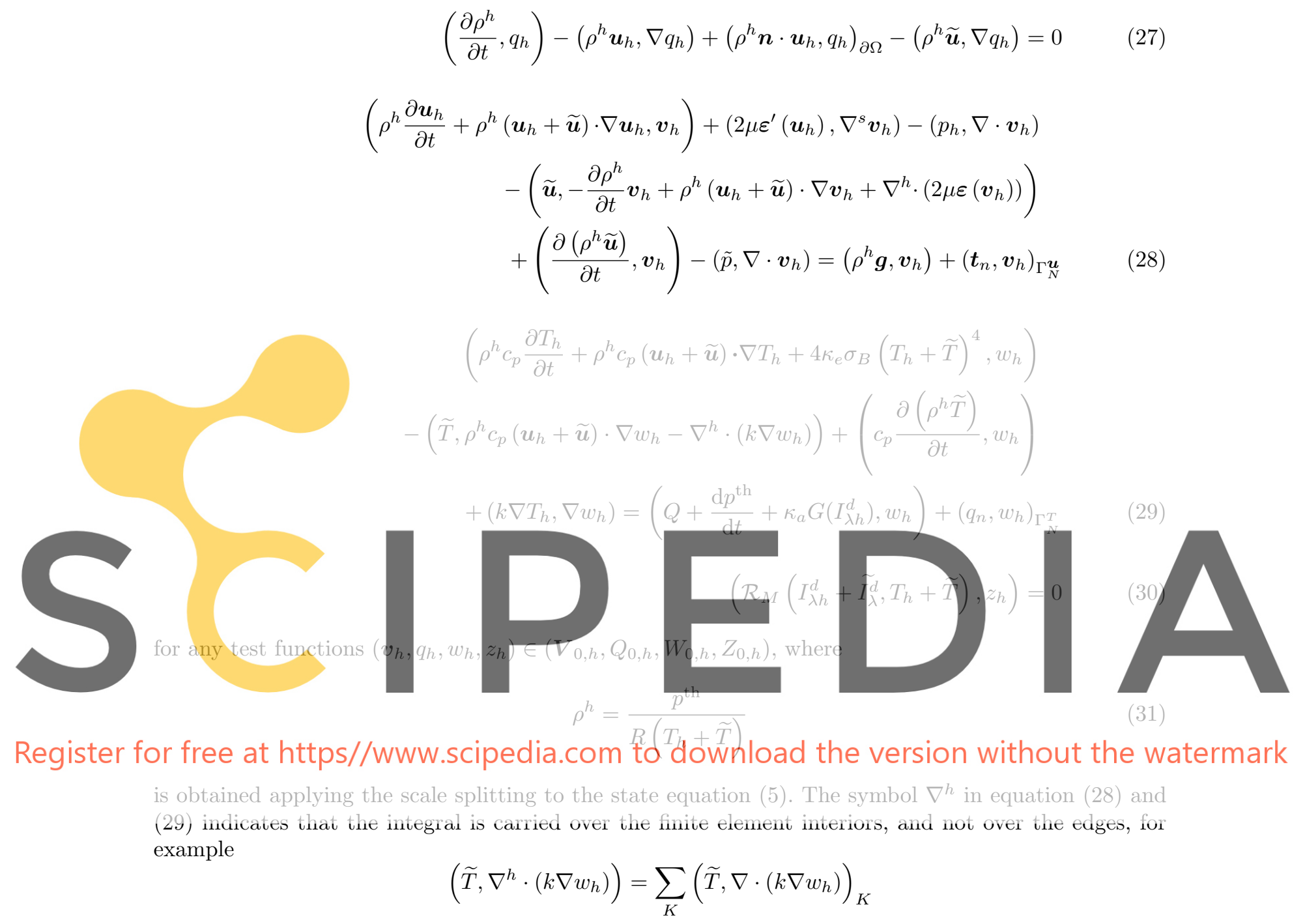

where $(\cdot, \cdot)_{K}$ is the $L^{2}(K)$ inner product.

Remark 1 The radiation intensity subscale in (30) depends on the particular directional discretization used. As described in Section 2.2, our goal is to present a stabilization method independent of the directional discretization in the radiation transport equation. A complete discussion on the approximation of the radiation intensity subscale when the DOM method is used can be found in [1]. In turn, when, e.g., the P1 method is used, the resulting problem is elliptic and one can simply consider $\widetilde{I_{\lambda}^{d}}=0$. As this choice only affects the approximation of the radiation transport equation and not the coupling with the hydrodynamic problem we will consider $\widetilde{I_{\lambda}^{d}}=0$ in what follows. It is worth noting that the numerical results obtained using the DOM method presented in Section 6 were obtained using the approximation discussed in [1].

Remark 2 The temperature has been split into $T_{h}+\widetilde{T}$ inside the radiative operator $\mathcal{R}_{M}$. If the temperature subscale is neglected in (30) it is convenient to neglect it also in the energy equation (29). Otherwise energy will not be globally conserved, as it will be shown in Section 4.

Remark 3 It is known that the absorption coefficient $\kappa_{a}$ depends strongly on temperature, being correlated with the thermal radiation emission $I_{b}$ and the radiation field $I$. It is very important to model these correlations, especially in combustion problems because temperature is highly fluctuating in space 
and time. When the temperature scale splitting is taken into account inside the radiative model, the absorption and emission coefficients $\kappa_{a}$ and $\kappa_{e}$ should be evaluated in terms of $T_{h}+\widetilde{T}$ through Eqs. (9) and (10). The correlations between temperature and blackbody radiation $I_{b}$, and temperature and the radiative field $I$ are modeled when computing $\kappa, G$ and $I_{b}$ in terms of $T_{h}+\widetilde{T}$. This is only done when introducing $T_{h}+\widetilde{T}$ in the radiative model.

The nonlinear scale splitting of the convective terms (in the momentum and energy equations) permits to model turbulence without the use of any physical model. The behavior of this numerical method in the large eddy simulation of thermally coupled turbulent flows at low Mach number is analyzed in [2]. The extension of the method to radiative flows leads to the (temperature) scale splitting of the nonlinear radiative terms. These terms are thought to model the physical subgrid behavior that cannot be captured by the mesh, and therefore to improve the obtained solutions of the equations. We have observed in numerical examples that the consideration of the temperature scale splitting $T_{h}+\widetilde{T}$ in the energy and radiation equations increases the radiative heat flux from hot zones, being this effect peculiar of turbulence-radiation interaction models, as explained in [6].

In order to give a closure to system (27)-(31) we need to define how the subscales $\widetilde{u}, \widetilde{p}$ and $\widetilde{T}$ are computed. In the same way the finite element equations can be understood as the projection of the original equations onto the finite element spaces $\boldsymbol{Y}_{h}$, the equations for the subscales are obtained by projecting the original equations onto their corresponding spaces $\widetilde{\boldsymbol{Y}}$. The hydrodynamic subscale equations are written as
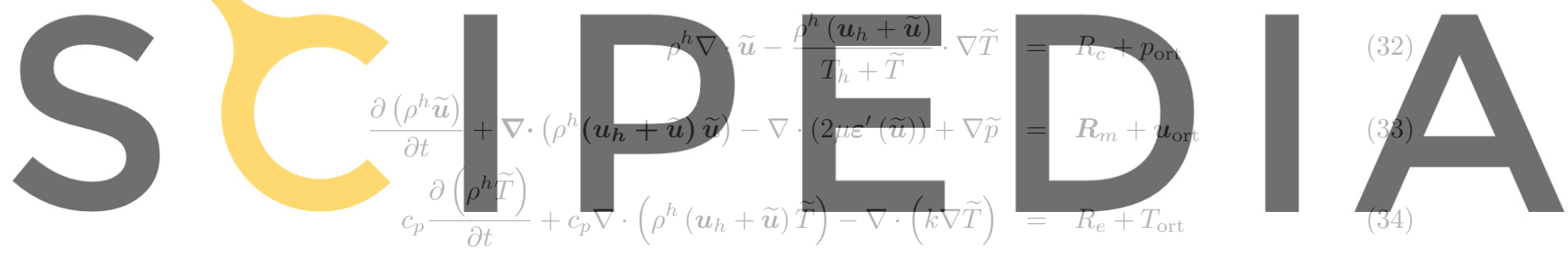

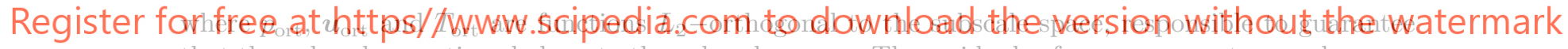

that the subscale equations belong to the subscale spaces. The residuals of mass, momentum and energy equations are respectively

$$
\begin{gathered}
R_{c}=-\frac{\partial \rho^{h}}{\partial t}-\rho^{h} \nabla \cdot \boldsymbol{u}_{h}+\frac{\rho^{h}\left(\boldsymbol{u}_{h}+\widetilde{\boldsymbol{u}}\right)}{T_{h}+\widetilde{T}} \cdot \nabla T_{h} \\
\boldsymbol{R}_{m}=\rho^{h} \boldsymbol{g}-\rho^{h} \frac{\partial \boldsymbol{u}_{h}}{\partial t}-\rho^{h}\left(\boldsymbol{u}_{h}+\widetilde{\boldsymbol{u}}\right) \cdot \nabla \boldsymbol{u}_{h}+\nabla \cdot\left(2 \mu \varepsilon^{\prime}\left(\boldsymbol{u}_{h}\right)\right)-\nabla p_{h} \\
R_{e}=Q+\frac{\mathrm{d} p^{\mathrm{th}}}{\mathrm{d} t}-\rho^{h} c_{p} \frac{\partial T_{h}}{\partial t}-\rho^{h} c_{p}\left(\boldsymbol{u}_{h}+\widetilde{\boldsymbol{u}}\right) \cdot \nabla T_{h}+\nabla \cdot\left(k \nabla T_{h}\right) \\
\quad+\kappa G-4 \kappa_{e} \sigma_{B}\left(T_{h}+\widetilde{T}\right)^{4}
\end{gathered}
$$

Approximation of the subscales We will approximate the subscale problem replacing the (spatial) differential operators of mass, momentum and energy equations by the algebraic operators $\tau_{c}^{-1}, \tau_{m}^{-1}$ and $\tau_{e}^{-1}$, respectively. The approximation to the subscale equations (32)-(34) within each element of the finite element partition reads

$$
\begin{aligned}
\frac{1}{\tau_{c}} \widetilde{p} & =R_{c}+p_{\text {ort }}=R_{c}^{\prime} \\
\frac{\partial\left(\rho^{h} \widetilde{\boldsymbol{u}}\right)}{\partial t}+\frac{1}{\tau_{m}} \widetilde{\boldsymbol{u}} & =\boldsymbol{R}_{m}+\boldsymbol{u}_{\mathrm{ort}}=\boldsymbol{R}_{m}^{\prime} \\
c_{p} \frac{\partial\left(\rho^{h} \widetilde{T}\right)}{\partial t}+\frac{1}{\tau_{e}} \widetilde{T} & =R_{e}+T_{\text {ort }}=R_{e}^{\prime}
\end{aligned}
$$


The stabilization parameters $\tau_{c}, \tau_{m}$ and $\tau_{e}$ are defined as

$$
\begin{aligned}
\tau_{c} & =\frac{h^{2}}{c_{1} \rho^{h} \tau_{m}}=\frac{\mu}{\rho^{h}}+\frac{c_{2}}{c_{1}}\left|\boldsymbol{u}_{h}+\widetilde{\boldsymbol{u}}\right| h \\
\tau_{m} & =\left(c_{1} \frac{\mu}{h^{2}}+c_{2} \frac{\rho^{h}\left|\boldsymbol{u}_{h}+\widetilde{\boldsymbol{u}}\right|}{h}\right)^{-1} \\
\tau_{e} & =\left(c_{1} \frac{k}{h^{2}}+c_{2} \frac{\rho^{h} c_{p}\left|\boldsymbol{u}_{h}+\widetilde{\boldsymbol{u}}\right|}{h}\right)^{-1}
\end{aligned}
$$

where $h$ is the element size and $c_{1}$ and $c_{2}$ are algorithmic constants whose values for linear elements are $c_{1}=4$ and $c_{2}=2$ in the numerical examples. It is important to remark that (39) and (40) are nonlinear equations. A deeper insight about the present subscale model can be found in [3], where also some linearization schemes are presented.

Remark 4 The spatial-differential operator in the subscale equation (34) has been approximated to obtain Eq. (40). Note however, that $\widetilde{T}$ still appears in the right hand side of Eq. (40) in the nonlinear reactive term $\kappa_{e} \sigma_{B}\left(T_{h}+\widetilde{T}\right)^{4}$. In the case of a linear convection-diffusion-reaction equation the reactive term is kept on the left hand side and a stabilization parameter of the form
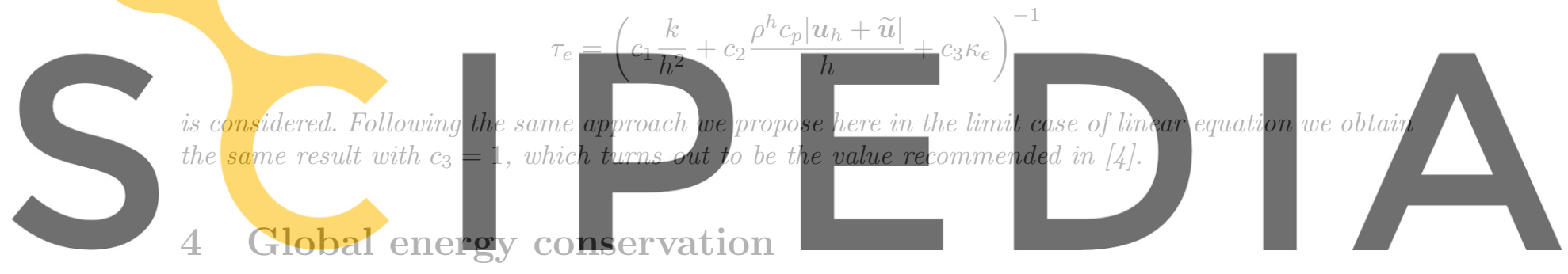

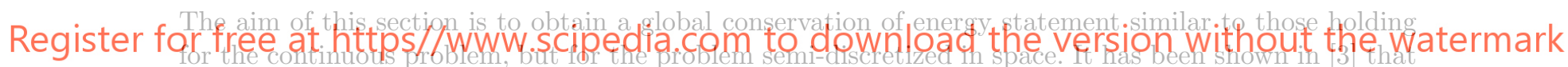

global conservation statements for mass, momentum and energy without radiative terms hold when equal interpolation is used for all variables. We shall see that the total energy will be globally conserved only if the radiative model $\mathcal{R}_{M}$ conserves radiation energy.

When the radiation transfer equation (4) is integrated over all solid angle directions, and over all the spectral domain $\lambda \in \mathbb{R}^{+}$, the zeroth moment radiative equation is obtained:

$$
\nabla \cdot \boldsymbol{q}_{r}-\kappa_{a} G=4 \kappa_{e} \sigma_{B} T^{4}
$$

This equation is satisfied by most radiative models that can be understood as angular discretization, as DOM and $P_{1}$. After integration of this equation over all the spatial domain $\Omega$, the following radiation conservation statement for the continuous problem is obtained

$$
\int_{\Omega}\left(4 \kappa_{e} \sigma_{B} T^{4}-\kappa_{a} G\right) d \Omega=\int_{\partial \Omega} \boldsymbol{q}_{r} \cdot \boldsymbol{n} \mathrm{d} \Gamma
$$

When the discrete counterpart of this conservation statement is satisfied by the discrete approximation of radiative models $\mathcal{R}_{M}$ (30), it is said that the radiation model conserves radiation energy. This is the case of the finite element approximation of the DOM and the $P_{1}$ method, which read

$$
\int_{\Omega}\left(4 \kappa_{e} \sigma_{B}\left(T_{h}+\widetilde{T}\right)^{4}-\kappa_{a} G\right) d \Omega=\int_{\partial \Omega} \boldsymbol{q}_{r} \cdot \boldsymbol{n} \mathrm{d} \Gamma
$$

Let us consider the finite element space for the temperature equation without Dirichlet boundary conditions, and an augmented problem that also contains the fluxes at the Dirichlet boundaries as unknowns [10]. When using equal interpolation spaces for the temperature and pressure equations $\left(W_{h}=\right.$ 
$Q_{h}$ ), it can be shown that taking the test function $w_{h}=1$ in (the augmented problem corresponding to) the finite element energy Eq. (29), and replacing (45) we get the relation

$$
\int_{\Omega} c_{p} \frac{\partial}{\partial t}\left(\rho^{h}\left(T_{h}+\widetilde{T}\right)\right) \mathrm{d} \Omega=\int_{\Omega}\left(Q+\frac{\mathrm{d} p^{\mathrm{th}}}{\mathrm{d} t}\right) \mathrm{d} \Omega-\int_{\partial \Omega}\left(q_{n}+\boldsymbol{n} \cdot\left(\boldsymbol{q}_{r}+\boldsymbol{u}_{h} \rho^{h} c_{p} T_{h}\right)\right) \mathrm{d} \Gamma
$$

which is the discrete counterpart of energy conservation equation (3) integrated over domain $\Omega$. Therefore, Eq. (46) implies energy conservation. For ideal gases the internal energy per unit mass is $e=c_{v} T$, where $c_{v} \equiv c_{p} / \gamma$. According to that, we define at the discrete level the discrete internal energy per unit volume as $\rho^{h} e^{h}=\rho^{h} c_{v}\left(T_{h}+\widetilde{T}\right)$. Replacing this definition in Eq. (46), after some operations (see [3]), we arrive to the first law of thermodynamics for open systems in terms of the internal energy:

$$
\int_{\Omega} \frac{\partial\left(\rho^{h} e_{h}\right)}{\partial t} \mathrm{~d} \Omega=\int_{\Omega} Q \mathrm{~d} \Omega+\int_{\partial \Omega}\left(q_{n}+q_{r n}-\boldsymbol{n} \cdot \boldsymbol{u}_{h}\left(\rho^{h} e^{h}+p^{\mathrm{th}}\right)\right) \mathrm{d} \Gamma
$$

where $q_{r n}=q_{r} \cdot n$ is the radiative heat flux leaving the domain. This equation indicates that the change in internal energy of the system is equal to the heat power added to the system plus the work done over the system $\left(-\int_{\partial \Omega} \boldsymbol{n} \cdot \boldsymbol{u}_{h} p^{\text {th }} \mathrm{d} \Gamma=-p^{t h} \int_{\Omega} \nabla \cdot \boldsymbol{u}_{h} \mathrm{~d} \Omega\right)$ plus the boundary fluxes of heat and internal energy, $q_{n}+q_{r n}$ and $n \cdot u_{h} \rho^{h} e^{h}$

To satisfy the energy conservation statement (46), and therefore (47), the discrete radiative model (30) must conserve the discrete radiation energy (statement (45)). The discrete radiation equation must depend on the temperature scale splitting $T_{h}+\widetilde{T}$ to be consistent with the temperature scale splitting in the energy equation, to achieve the energy conservation statement (45). If this splitting is ignored in
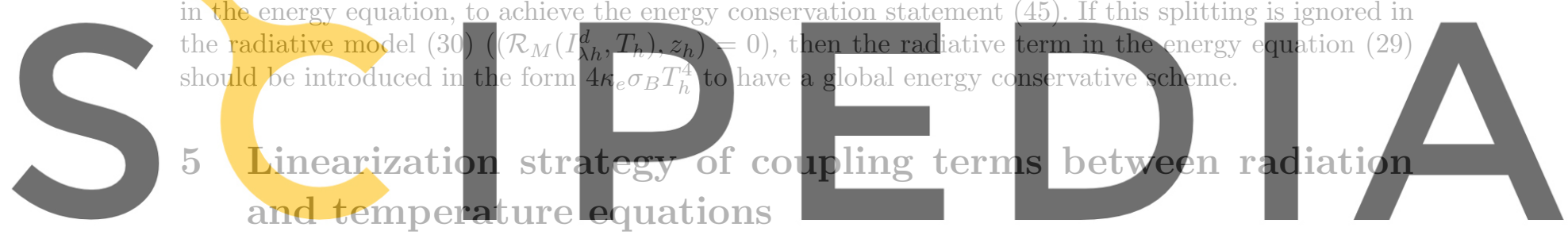

Register forf The coupling hetween, radiation and andergy equationg is extremely monlinear, and careful linearization

linearization of the terms involved in radiation. Linearization schemes of mass, momentum and energy equations without considering radiative coupling are explained in detail in [3].

Linearization of radiative terms in the finite element equation We will focus on how to linearize the energy equation (29); the radiative equation (30) is supposed to be solved in segregated form. The subscales are supposed to be given as we need to solve the finite element problem (29). The linearization of the radiative term $4 \kappa_{e} \sigma_{B}\left(T_{h}+\widetilde{T}\right)^{4}$ in the energy equation using a Newton Rapshon scheme is always convergent, because this term never changes its convexity (second derivative sign respect to $T_{h}$ remains unchanged). If the temperature $T_{h}$ is known at iteration $k$, the nonlinear term is linearized at iteration $k+1$ in terms of $T_{h}^{k+1}$, using a Newton Raphson scheme as

$$
4 \kappa_{e} \sigma_{B}\left(T_{h}^{k+1}+\widetilde{T}\right)^{4} \approx 4 \kappa_{e} \sigma_{B}\left(T_{h}^{k}+\widetilde{T}\right)^{3}\left(4 T_{h}^{k+1}-3 T_{h}^{k}+\widetilde{T}\right)
$$

Other linearization strategies were implemented, like the fixed point iteration

$$
4 \kappa_{e} \sigma_{B}\left(T_{h}^{k+1}+\widetilde{T}\right)^{4} \approx 4 \kappa_{e} \sigma_{B}\left(T_{h}^{k}+\widetilde{T}\right)^{3}\left(T_{h}^{k+1}+\widetilde{T}\right)
$$

or the following

$$
4 \kappa_{e} \sigma_{B}\left(T_{h}^{k+1}+\widetilde{T}\right)^{4} \approx 4 \kappa_{e} \sigma_{B}\left(T_{h}^{k}+\widetilde{T}\right)^{3}\left(2 T_{h}^{k+1}-T_{h}^{k}+\widetilde{T}\right)
$$

These iterations schemes diverged in many examples. The Newton Raphson scheme (48), besides being unconditionally convergent, converged always faster than the other linearization schemes.

We have solved the radiation equation (30) segregated from the temperature equation (29). The coupling between radiation and temperature equations has been found to converge much faster when applying an over-relaxation to temperature. 
Linearization of radiative terms in the subscale equation The subscale equations form a nonlinear system of equations that must be linearized. Linearization schemes for the subscale equations without considering radiation coupling are detailed in [3], where it was shown that the Newton-Raphson scheme applied to the monolithically coupled system of equations was the most efficient one. Radiative transfer introduces the nonlinear term $4 \kappa_{e} \sigma_{B}\left(T_{h}+\widetilde{T}\right)^{4}$ in the subscale energy equation (34). The Newton-Raphson scheme for the linearization of this radiative term inside the subscale energy equation (34) is (again) unconditionally convergent, because the function does not change its convexity. The finite element unknowns are assumed to be given as we need to solve the subscale problem. If the temperature subscale is known at iteration $k$, the linearization of the nonlinear reactive term with respect to $\widetilde{T}^{k+1}$ at iteration $k+1$ is

$$
4 \kappa_{e} \sigma_{B}\left(T_{h}+\widetilde{T}^{k+1}\right)^{4} \approx 4 \kappa_{e} \sigma_{B}\left(T_{h}+\widetilde{T}^{k}\right)^{3}\left(T_{h}+4 \widetilde{T}^{k+1}-3 \widetilde{T}^{k}\right)
$$

We obtained satisfactory and fast convergent results using this scheme.

Linearization of the coupled energy boundary condition When we want to impose an amount of conductive plus radiative heat flux through the boundaries, $q_{n}+q_{r n}=\mathcal{H}$, we need to apply the nonlinear boundary condition (17) to the energy equation (29). This condition couples temperature and radiation intensity on the boundary, affecting significantly the solution for optically thin problems, in which $\kappa_{a} L<1$, where $L$ is a characteristic length of the problem. We have found in the numerical experiments that a proper linearization of this boundary condition needs to be applied to achieve convergence. The most efficient method was to apply a Newton-Raphson scheme. Although it is always convergent, the solution converged extremely slowly when solving optically thin problems. When applying simpler linearization schemes the solution did not converge.

Assuming a known temperature at iteration $k$, boundary condition (17) is linearized to approximate temperature at iteration $k+1$ as

$$
-\boldsymbol{n} \cdot k \nabla T_{h}^{k+1}-\epsilon \sigma_{B}\left(T_{h}^{k}\right)^{3}\left(4 T_{h}^{k+1}-3 T_{h}^{k}\right)=\mathcal{H}-(1-r) H
$$

The resulting linearized boundary condition is of Robin type.

\section{An application example: fire in a 3D room with an open door}

This test example is a fire compartment similar to that considered in [3], but now simulating the effect of radiative heat transfer. In order to increase radiative heat transfer effects, the power of the heat source that models the fire has been increased.

The problem domain is $\Omega=[0, L] \times[0, L] \times[0, H]$ where $L=2.8 \mathrm{~m}$ and $H=2.18 \mathrm{~m}$. The compartment has an open door on the side wall of the room $(x=L)$ whose dimension is $0.7 \times 1.853 \mathrm{~m}^{2}$. A scheme of the problem domain is shown in Fig. 1. The fire is modeled by an uniform heat source of $30 \mathrm{~kW}$, located at the center of the room just over the floor, with dimensions $0.84 \times 0.84 \times 0.218 \mathrm{~m}^{3}$. Adiabatic boundary conditions are imposed on all the walls. Non slip boundary conditions for velocity are imposed on all the boundaries except the door, where atmospheric boundary condition is imposed, that is, a traction $\boldsymbol{t}_{n}=(-\rho|\boldsymbol{g}| z, 0,0)$. As the flow is open $\left(\left(\Gamma_{N}^{\boldsymbol{u}} \neq \emptyset\right)\right)$, the thermodynamic pressure is set constant in time to $p^{\text {th }}=101325 \mathrm{~Pa}$. The initial temperature and velocity values are $T_{0}=300 \mathrm{~K}$ and $\boldsymbol{u}_{0}=\mathbf{0}$ over all domain $\Omega$. Furthermore, the viscosity is $\mu=0.0094 \frac{\mathrm{kg}}{\mathrm{ms}}$ and $\operatorname{Pr}=0.71$. The gravity is set to $\boldsymbol{g}=(0,0,-9.8) \mathrm{m} / \mathrm{s}^{2}$. The medium is treated as a gray body, with homogeneous absorption and emissive coefficientes $\kappa_{a}=\kappa_{e}=10 \mathrm{~m}^{-1}$, and a zero scattering coefficient $\sigma_{s}=0$.

The radiative field was obtained using both the spherical harmonics $P_{1}$, and the discrete ordinates method (DOM) approximation to the radiative transport equation. For the DOM discretization we used the $S 10$ [12] set of ordinates and weights for DOM, consisting in 120 ordinates.

The compartment was meshed using a grid of $40 \times 40 \times 40$ uniform trilinear elements $Q_{1}$. We solved the problem using finite-difference time integration schemes, with uniform step size of $\delta t=1.0 \mathrm{~s}$. We compared the obtained solutions with three different stabilization methods, namely, the classical SUPG method, and the dynamical and nonlinear subscale method presented in this paper with and 


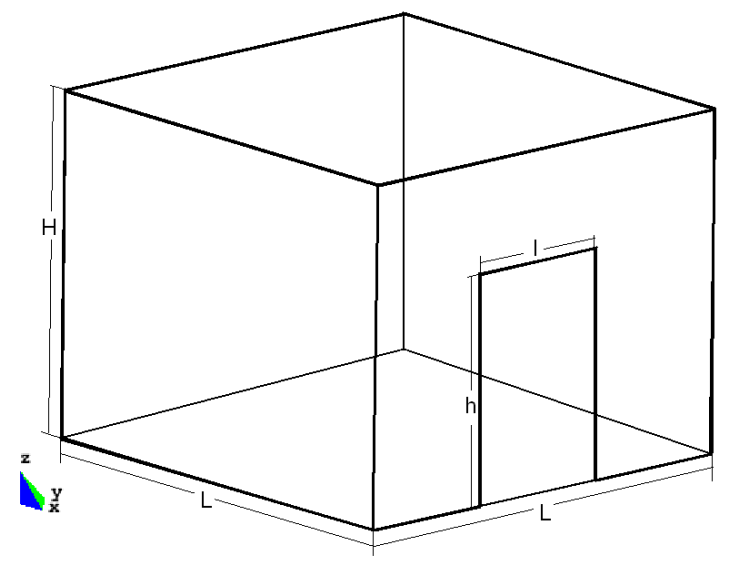

Figure 1: Problem domain of the room with an open door
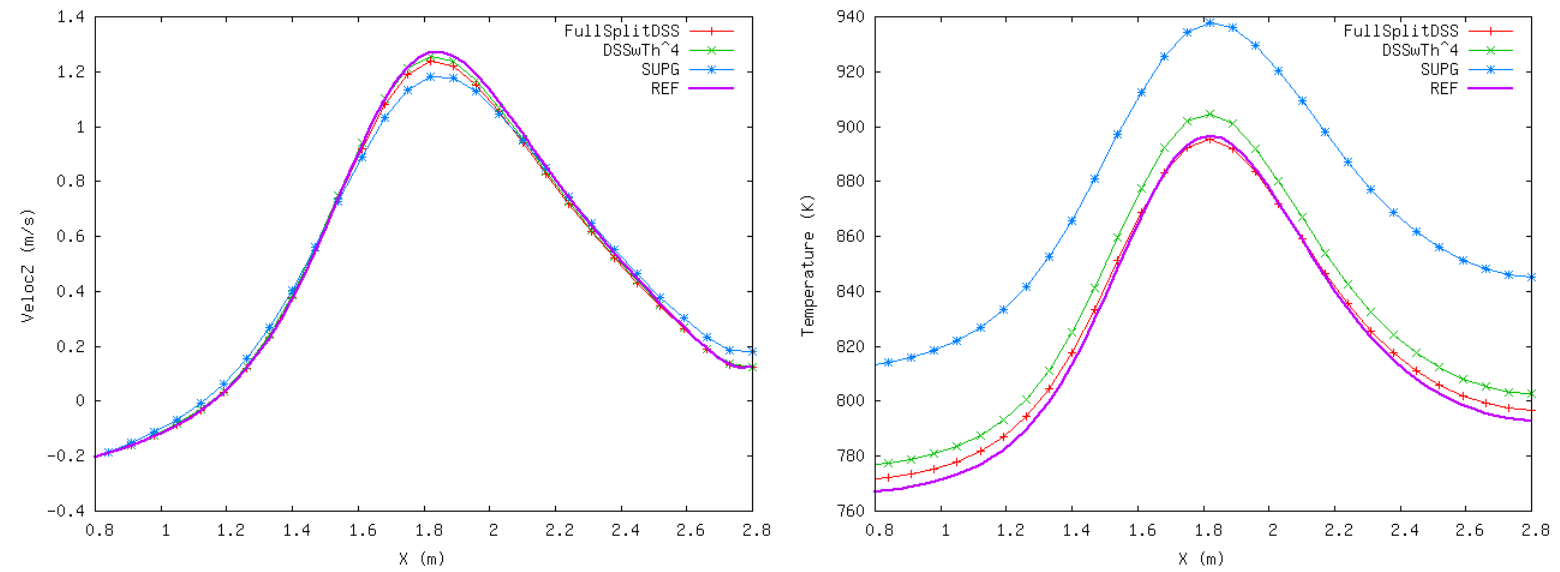

Figure 2: Temperature and vertical velocity distributions along the $x$ direction using the $P_{1}$ method

without temperature scale splitting of the radiative term, $4 \kappa \sigma_{B} T^{4}$. We call this method DSS in the following. When temperature scale splitting is (not) considered in the energy equation, then it is also (not) considered as radiative source in the radiation equation. The obtained results are compared against a reference solution obtained using the SUPG method over a fine mesh of $80 \times 80 \times 80$ uniform elements, and a time step size of $\delta t=0.5 \mathrm{~s}$, using the $P_{1}$ method and the DOM. The computation is advanced until $t_{\text {end }}=180.0 \mathrm{~s}$ using the second order time integration scheme BDF2. The tolerance for the nonlinear iterations was set to $5 \cdot 10^{-4}$ in the relative norm of the difference between two iterates.

In Figs. 2, 3 and 4 the temperature and vertical velocity distributions along the $x, y$ and $z$ directions using the DSS and SUPG methods at $t=t_{\text {end }}$ are shown for the $P_{1}$ method. The same results are shown when using the DOM in Figs. 5, 6 and 7 using the DOM. The DSS method is labeled as FullSplitDSS when the temperature subscale is kept in all terms, and it is labeled as DSSwTh ${ }^{\wedge} 4$ when the temperature subscale is not taken into account in the radiative term. In all those figures the greater similarity of the results with the reference solution when using the DSS method against SUPG method is clearly observed, in spite of the fact that the reference solution was obtained using the SUPG method on a finer mesh. The same observation was done in [3] for the problem without radiation. It is observed that performing the temperature scale splitting in the radiative term has the effect of decreasing the obtained temperature, closer to the reference solution. The effect of the temperature splitting is more noticeable in the temperature solution; the vertical velocity is less affected, and it is difficult to conclude if there exists an improvement.

The time evolution of temperature and vertical velocity (along the $z$ direction) at points of coordi- 

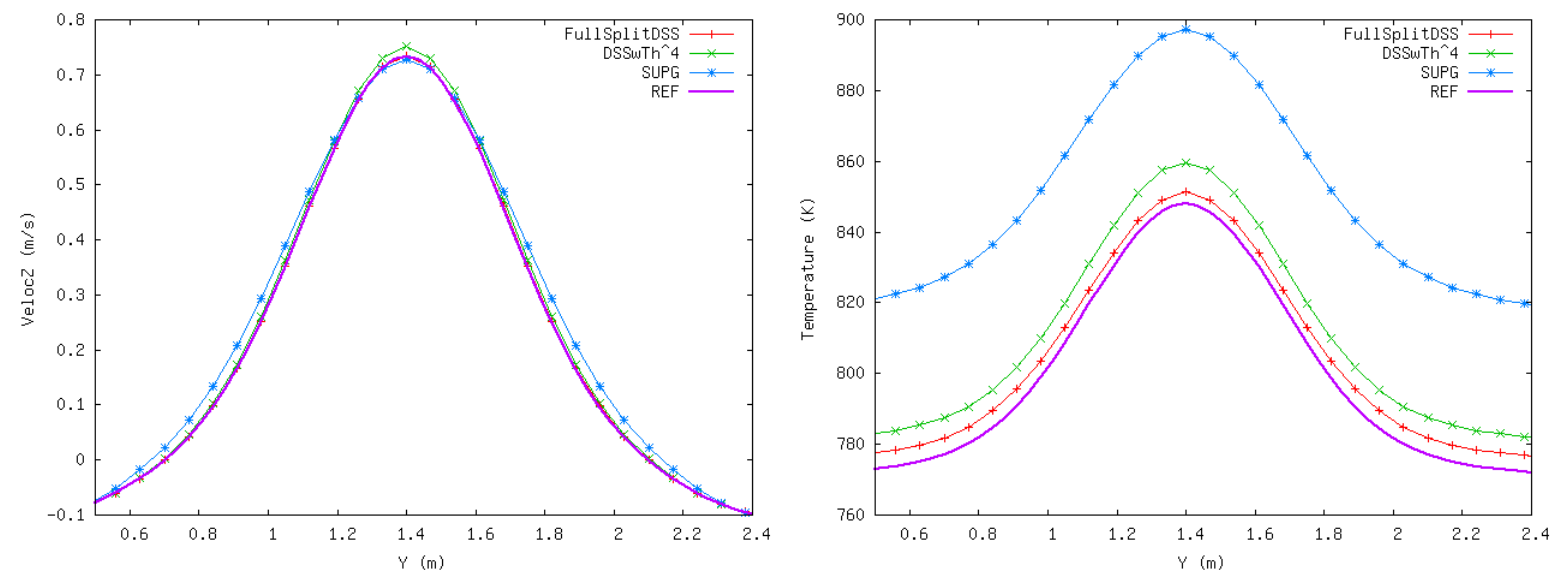

Figure 3: Temperature and vertical velocity distributions along the $y$ direction using the $P_{1}$ method
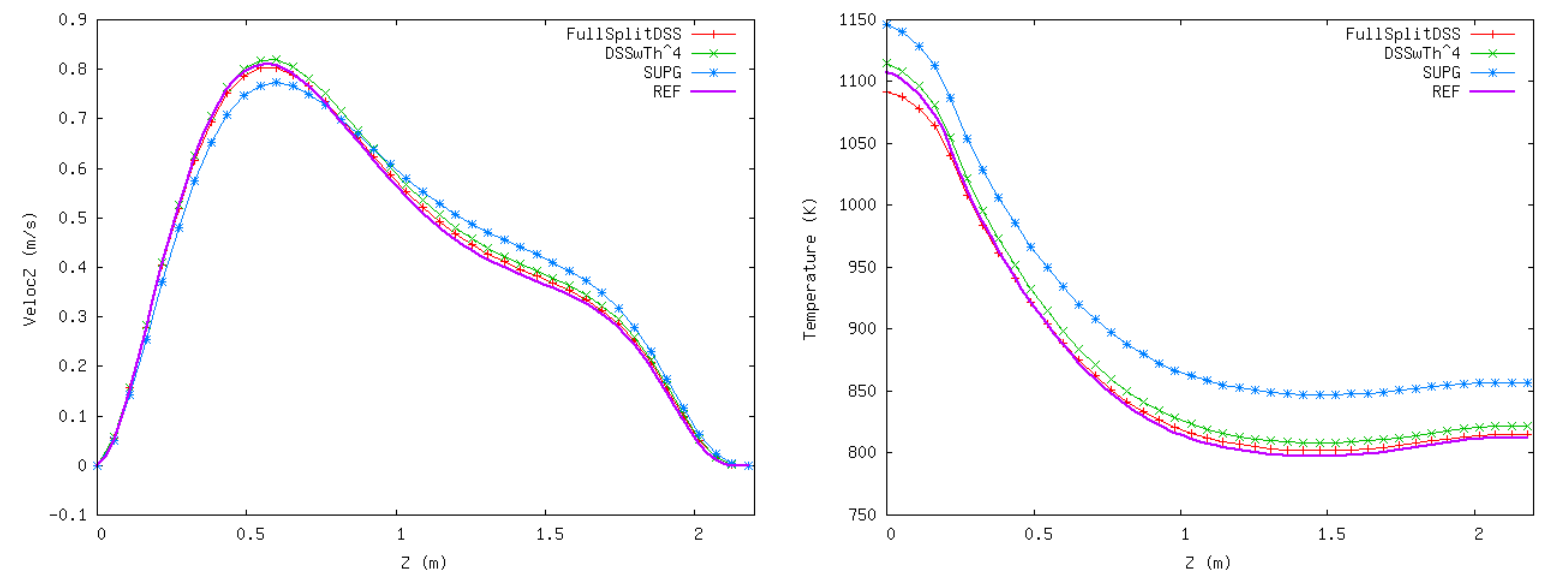

Figure 4: Temperature and vertical velocity distributions along the $z$ direction using the $P_{1}$ method

nates $(1.55,1.4,0.55),(1.3,1.3,0.2)$ and $(1.4,1.4,1.0)$ (in meters) are compared in Figs. $8,9,10$ for the $P_{1}$ method, and in Figs. 11, 12 and 13 for the DOM. It is observed that when using the SUPG method the solution differs much more from the reference solution than when using the DSS method. It is difficult to observe the difference in the solutions when splitting or not the temperature in the radiative term, and it is even more difficult to confirm if there exist any improvement. However, observing temperature cuts at time $t_{\text {end }}$ it is seen that there exists such an improvement in temperature field.

In Fig. 16 the obtained temperature distribution along the $x$ and $y$ directions are compared when using the DOM and the $P_{1}$ methods. The small difference in the solution indicates that the $P_{1}$ method gives reasonable good solutions. This is due to the fact that the linearly anisotropic angular distribution of radiation intensity assumed by the $P_{1}$ method is a good approximation, since we are in an optically thick case $(\tau \approx 25)$.

In Fig. 14 the distribution of the temperature subscale over the plane $y=L / 2$ when $t=t_{\text {end }}$ is shown. It is seen that the maximum subscale values are located over the source term position, contributing to a raise of radiation source when adding the temperature subscale. We would like to mention that a spatial temperature subscale distribution over the source with mean value zero, and zero skewness, would contribute to a positive raise of the radiative source. In Fig. 15 the temperature distribution over the plane $y=L / 2$ at time $t=t_{\text {end }}$ when considering and not considering the temperature subscale in the radiative term of the energy equation are shown. The effect of considering the full scale splitting is a temperature decrease, a similar effect of radiation-turbulence interaction models. Fig. 15 shows the radiative heat distribution over the plane $y=L / 2$ at time $t=t_{\text {end }}$ when considering and not considering 

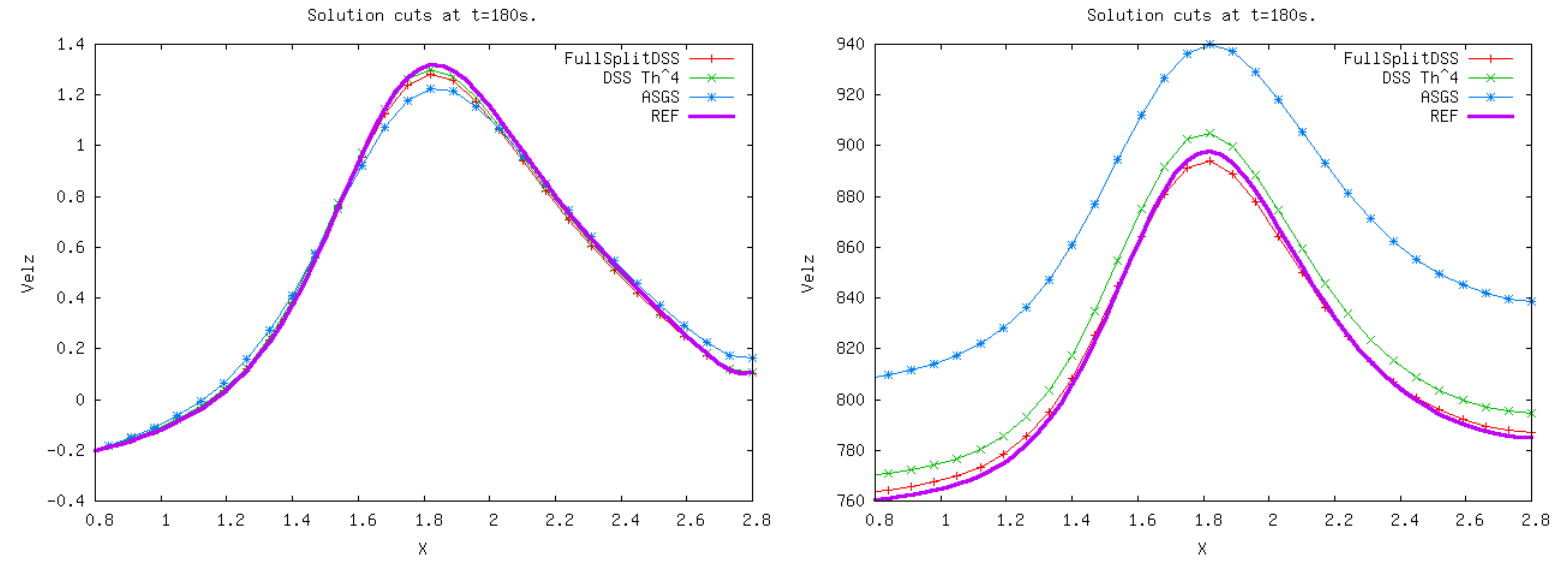

Figure 5: Temperature and vertical velocity distributions along the $x$ direction using the DOM
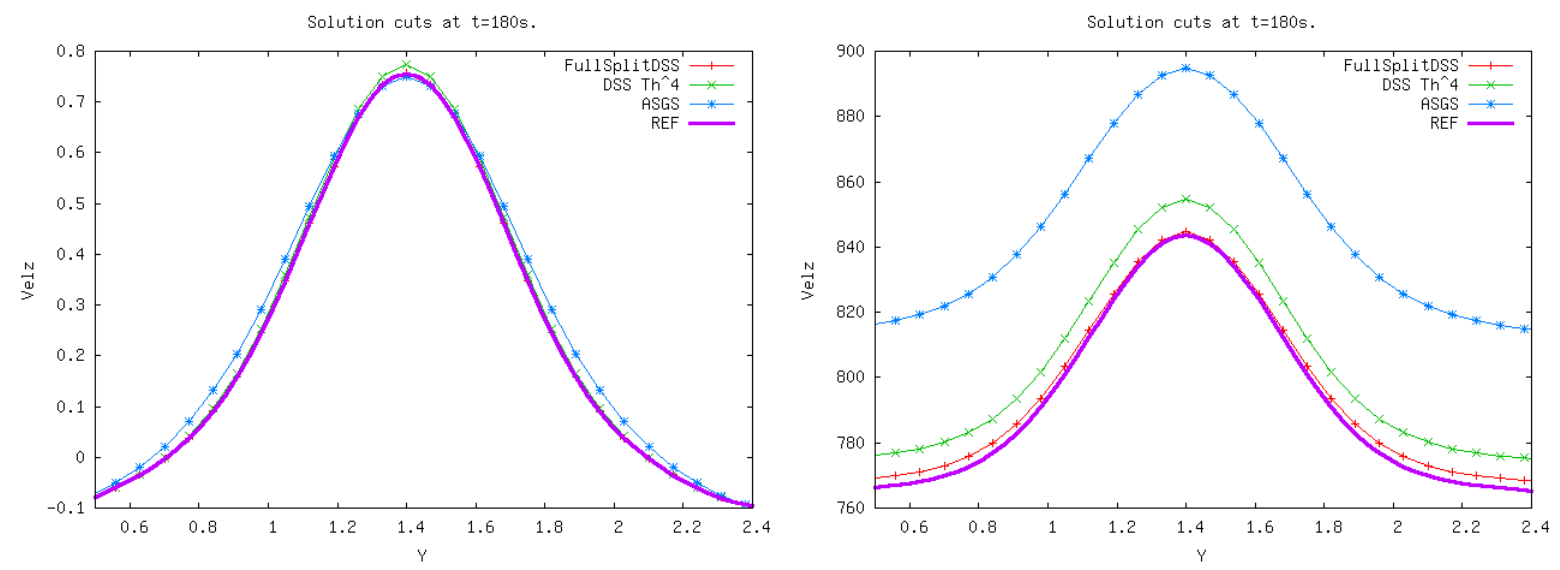

Figure 6: Temperature and vertical velocity distributions along the $y$ direction using the DOM

the temperature subscale in the radiative term of the energy equation. It is seen that the radiative heat flux decreases when considering the temperature splitting, which is due to the decrease of temperature over the fire.

The effect of considering temperature scale splitting in the radiative term $4 \kappa \sigma_{B}\left(T_{h}+\widetilde{T}\right)^{4}$ enhances the obtained results, increasing the radiative heat flux from hot zones. This is the expected turbulent effect of modeling a subgrid temperature. We believe that the difference in the results when considering the temperature subscale in the radiative term will be more noticeable in turbulent flames, with combustion models. We expect that for turbulent flows the effect of modeling the emitting and absorbing radiation effects as $4 \kappa_{e}(T) \sigma_{B}\left(T_{h}+\widetilde{T}\right)^{4}$, in a purely numerical form, will model the turbulent radiation interaction enhancing the obtained solution.

Performance of the methods The total number of nonlinear iterations needed to solve the problem (i.e. the sum of the nonlinear iterations performed in all time steps) and the total CPU time are indicated in Table 1 when using the $P_{1}$ method. The CPU time spent when using the DOM is much higher for the radiation problem, but it is similar for the low Mach number problem. The CPU time spent for the assembly of the low Mach equations (which includes numerical integration and solution of the subscale problem, i.e. operations involving a loop over integration points) and the CPU time spent in the (linear) solver procedures are also indicated. The CPU time spent solving the radiation equation is also indicated.

The total number of nonlinear iterations for the low Mach equations is $5 \%$ lower using the DSS method. However, the use of this method increases the CPU time of assembly operations per iteration, 

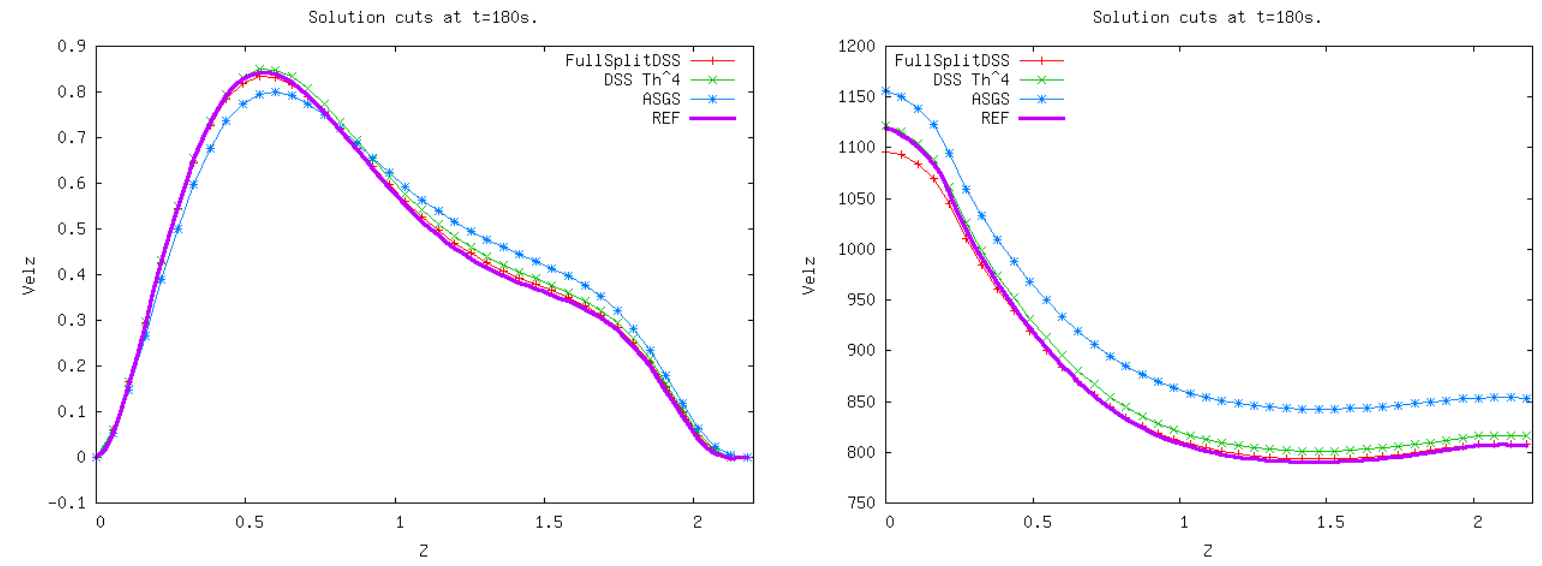

Figure 7: Temperature and vertical velocity distributions along the $z$ direction using the DOM
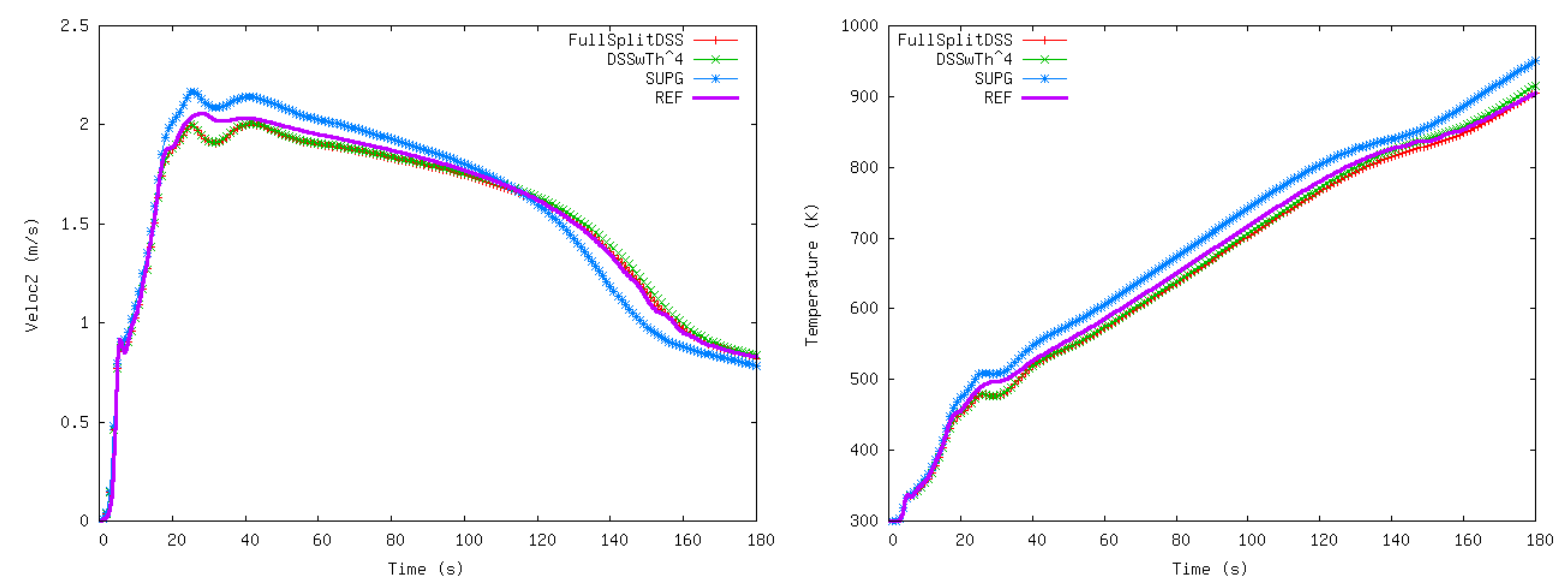

Figure 8: Time evolution of temperature and vertical velocity (i.e. $\left.u_{z}\right)$ at point $(1.55,1.4,0.55) \mathrm{m}$ using $P_{1}$ method

and therefore the total CPU time is $6.5 \%$ higher using DSS method, but the obtained solution is much better. The same behavior was observed in the same problem in [3], without radiation, using a smaller fire source and the same mesh with the same time step. The DSS method is observed to be very competitive. The CPU time spent solving the radiation equations does not differ more than $1 \%$ using the different methods in low Mach number equations. The CPU time of DSS method does not change when taking into account the temperature splitting in the radiative term. The CPU solver time is a little lower using the SUPG method, that is, the system is a little better conditioned. This is due to the spatial discontinuities of the subscales that worsens the matrix condition. This small extra CPU time when using DSS method occurs at expenses of obtaining a much better solution.

\section{Conclusions}

A finite element approximation of the low Mach number equations coupled with a radiative heat transfer model based on a splitting of the unknowns into finite element and unresolvable components has been developed. The main ingredients of the formulation are:

- To consider time dependent subscales.

- To keep the subscale components in all the nonlinear terms. 

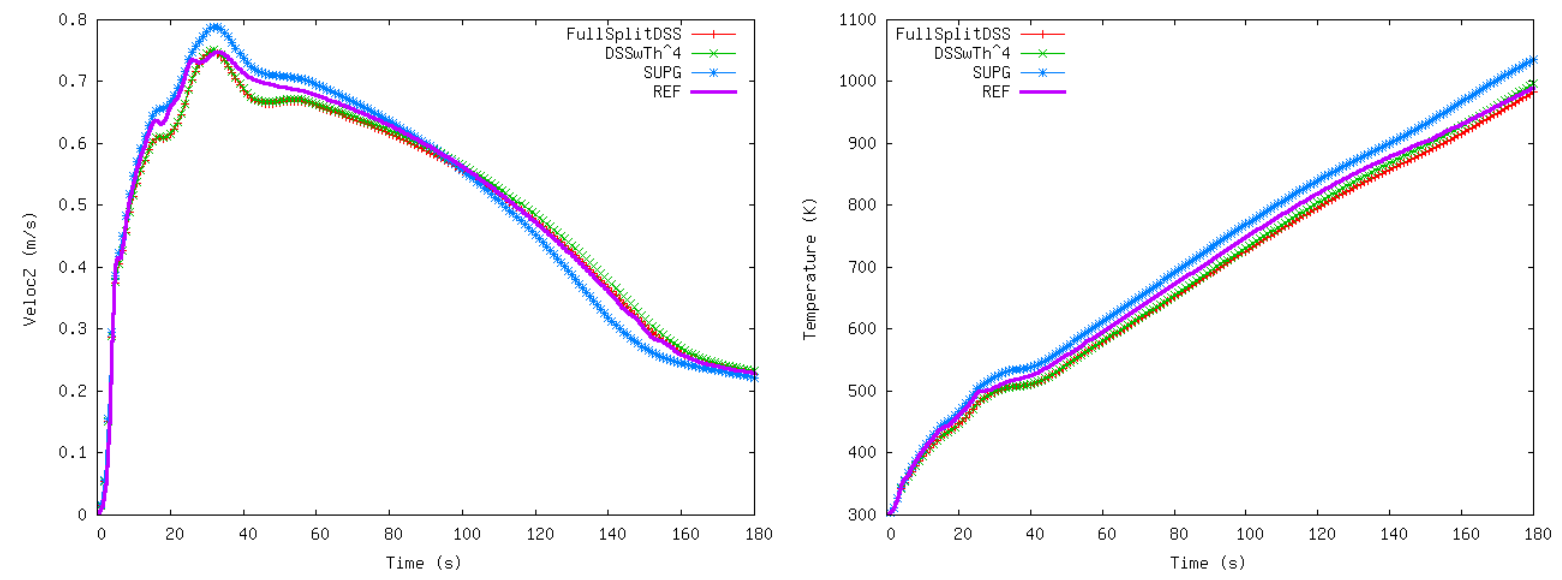

Figure 9: Time evolution of temperature and vertical velocity (i.e. $\left.u_{z}\right)$ at point $(1.3,1.3,0.2) \mathrm{m}$ using $P_{1}$ method
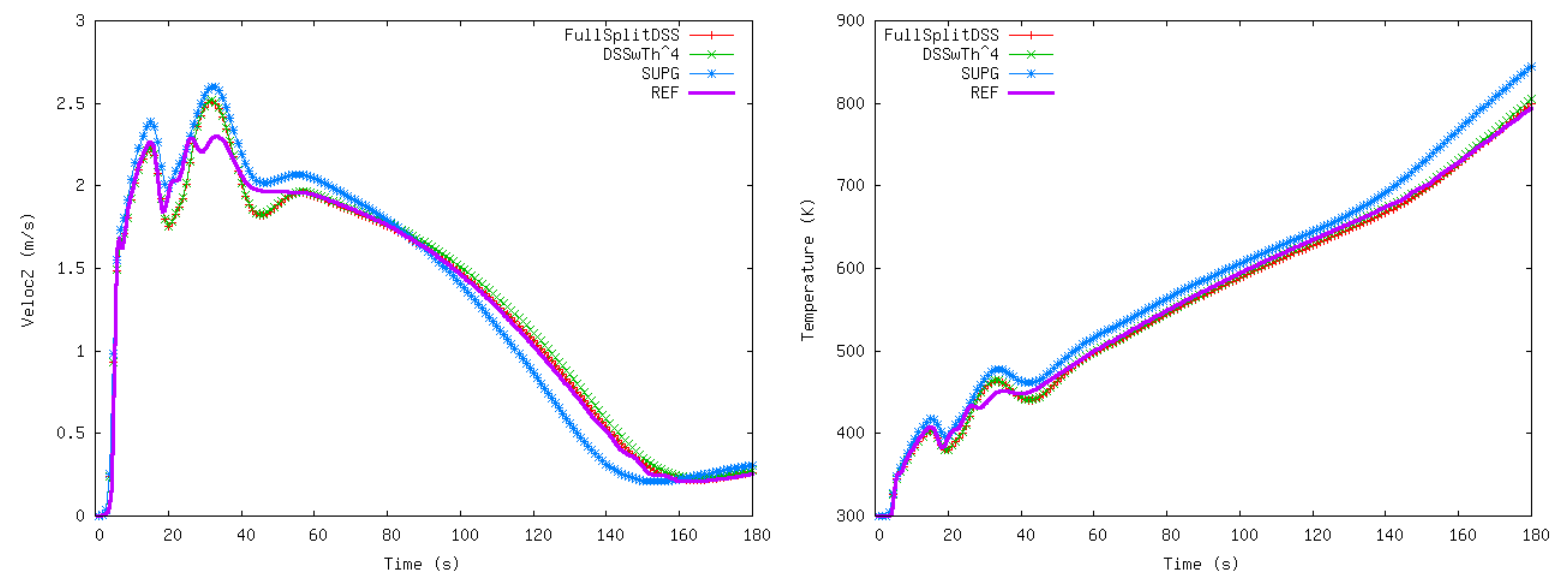

Figure 10: Time evolution of temperature and vertical velocity (i.e. $u_{z}$ ) at point $(1.4,1.4,1.0) \mathrm{m}$ using $P_{1}$ method

The effect of considering time dependent subscales is well known [5] and our experience with the low Mach number equations [3, 2] confirms the properties known for incompressible flows. The effect of considering the splitting of the unknowns in all the terms leads to a more accurate solution than classical stabilization methods and provides global mass, momentum and energy conservation when using equal interpolation spaces for the velocity, pressure and temperature equations. An improvement in the quality of the solution is obtained when considering the spliting of the radiation terms in the temperature equation.

We would like to stress, once again, that we keep the splitting of the unknowns in all terms also in the subscale equations, and we have numerically verified that this makes a difference in the accuracy of the scheme.

This nonlinear and transient treatment of the subscales has a computational cost, both in memory requirements and in CPU time. Nevertheless, the extra amount of memory needed only grows linearly with the number of nodes and will be usually dominated by the memory needed to solve the linear system and, as we have observed, the increase of CPU time in the DSS is very small.

The formulation intrinsically contains cross- and Reynolds- stress terms, and TRI terms that try to model the unsolved eddies and subgrid interaction between radiation and temperature, presenting an open door to turbulence modeling. The present method remains unchanged irrespective of whether laminar, transitional and turbulent situations are present. 

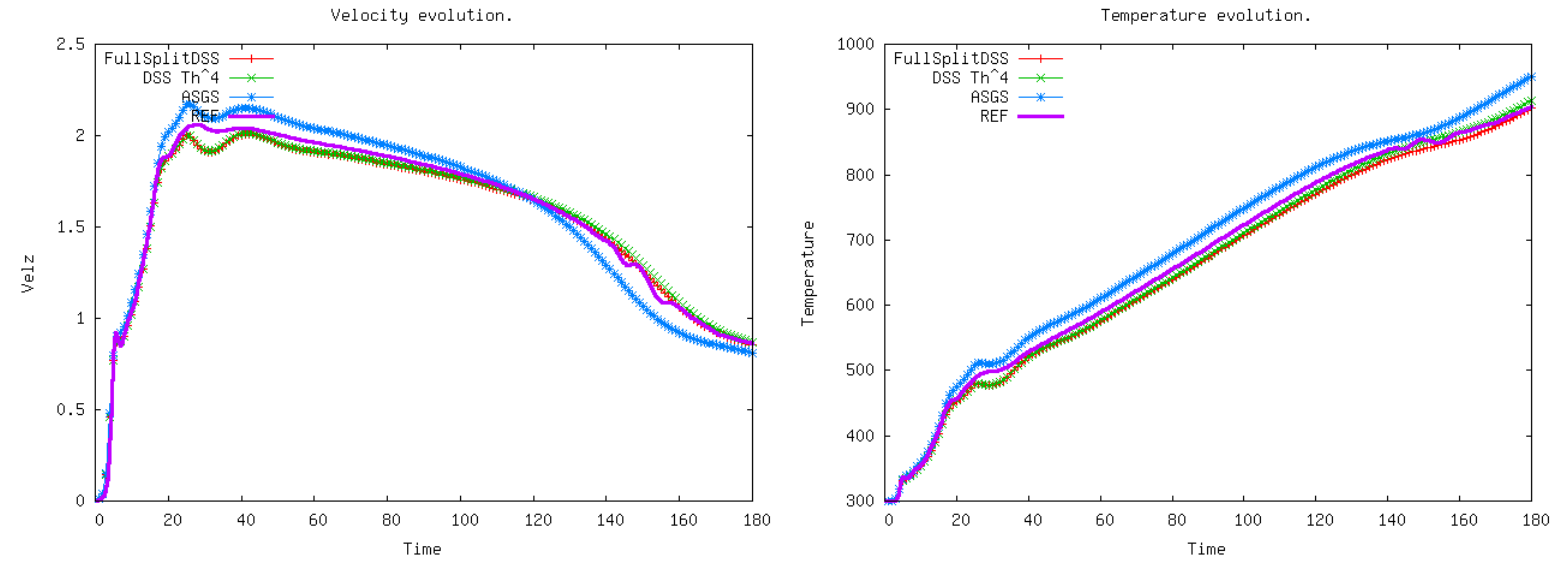

Figure 11: Time evolution of temperature and vertical velocity (i.e. $\left.u_{z}\right)$ at point $(1.55,1.4,0.55) \mathrm{m}$ using the DOM
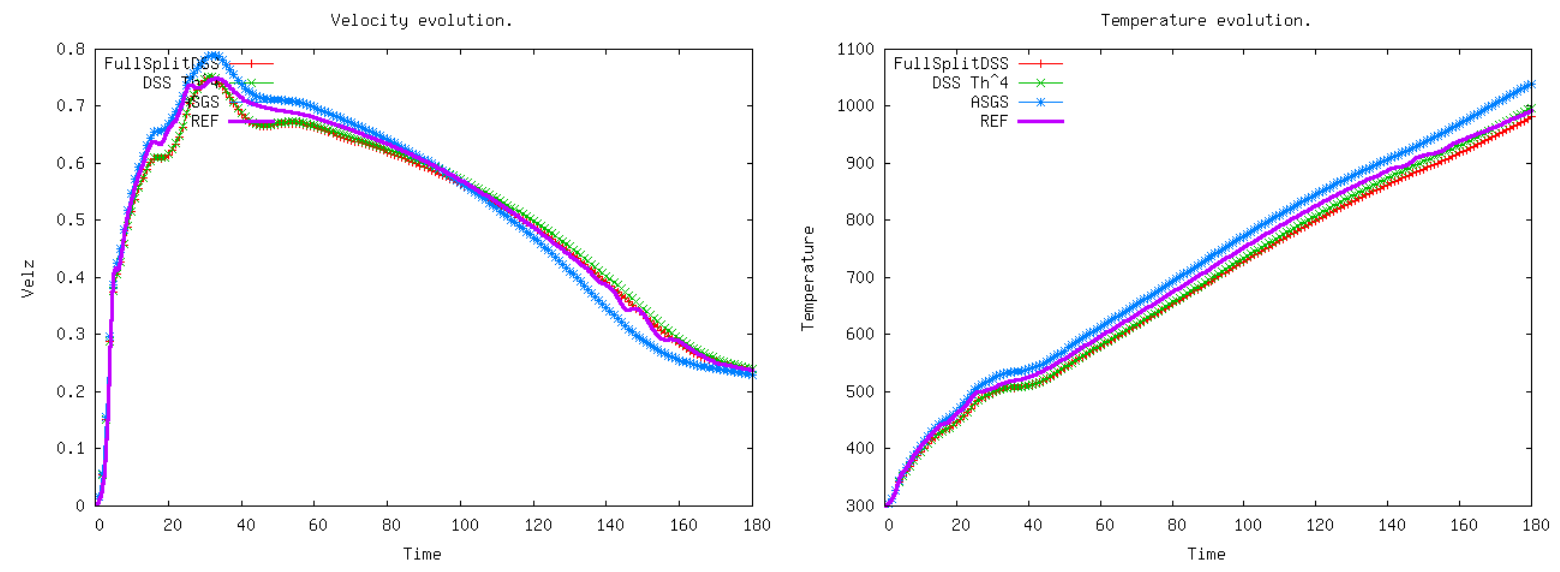

Figure 12: Time evolution of temperature and vertical velocity (i.e. $\left.u_{z}\right)$ at point $(1.3,1.3,0.2) \mathrm{m}$ using the DOM

We have emphasized the advantages of the temperature splitting $\left(T_{h}+\widetilde{T}\right)$ inside the radiative model and the radiative terms in the energy equation. When performing this splitting more accurate solutions were found in the numerical examples.

\section{Acknowledgments}

The authors acknowledge the financial support received from the Spanish Ministry of Economics and Competitiveness, National Programme of R\&D to the project PARANAT (ENE2011-28825). R. Codina also acknowledges the financial support received from the ICREA Acadèmia Program, from the Catalan Government.

\section{References}

[1] M. Avila, R. Codina, and J. Principe. Spatial approximation of the radiation transport equation using a subgrid-scale finite element method. Computer Methods in Applied Mechanics and Engineering, 200:425-438, 2011. 

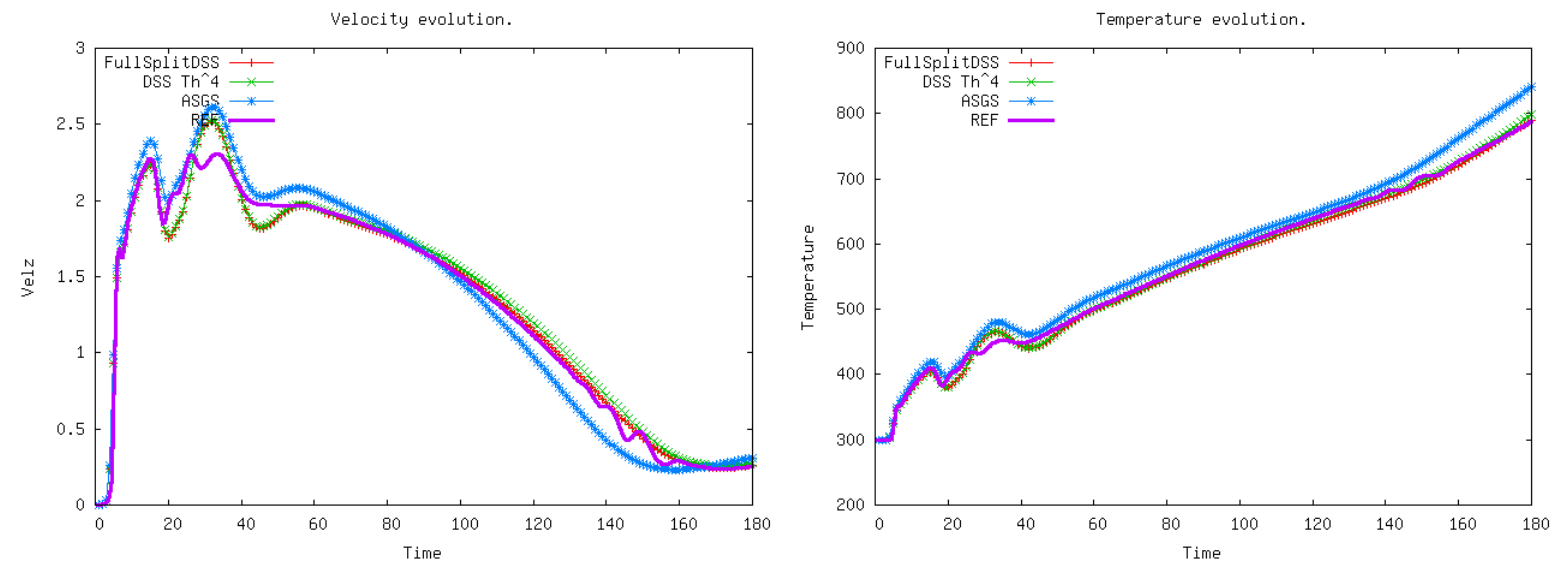

Figure 13: Time evolution of temperature and vertical velocity (i.e. $u_{z}$ ) at point $(1.4,1.4,1.0) \mathrm{m}$ using the DOM

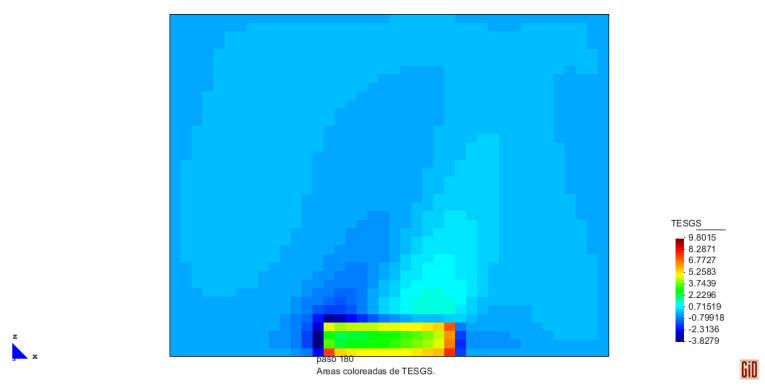

Figure 14: Temperature subscale distribution on the plane $y=L / 2$ )

[2] M. Avila, R. Codina, and J. Principe. Large eddy simulation of low Mach number flows using dynamic and orthogonal subgrid scales. Computers \& Fluids, 99:44-66, 2014.

[3] M. Avila, J. Principe, and R. Codina. A finite element dynamical nonlinear subscale approximation for the low Mach number flow equations. Journal of Computational Physics, 230:7988-8009, 2011.

[4] R. Codina. Comparison of some finite element methods for solving the diffusion-convection-reaction equations. Computer Methods in Applied Mechanics and Engineering, 156:185-210, 1998.

[5] R. Codina, J. Principe, O. Guasch, and S. Badia. Time dependent subscales in the stabilized finite element approximation of incompressible flow problems. Computer Methods in Applied Mechanics and Engineering, 196:2413-2430, 2007.

[6] P.J. Coelho. Numerical simulation of the interaction between turbulence and radiation in reactive flows. Progress in Energy and Combustion Science, 33:311-383, 2007.

\begin{tabular}{|c|c|c|c|c|c|c|}
\hline & \# iters. & total cpu(s) & cpu LM(s) & cpu Rad(s) & cpu assem LM(s) & cpu solv LM(s) \\
\hline \hline SUPG & 3579 & 82563 & 66182 & 16341 & 24159 & 42023 \\
\hline DSSwTh4 & 3438 & 88105 & 71896 & 16144 & 28359 & 43537 \\
\hline DSSFULL & 3406 & 88103 & 71985 & 16054 & 28549 & 43436 \\
\hline
\end{tabular}

Table 1: Comparison of the total number of iterations and the required cpu time for the different methods. 

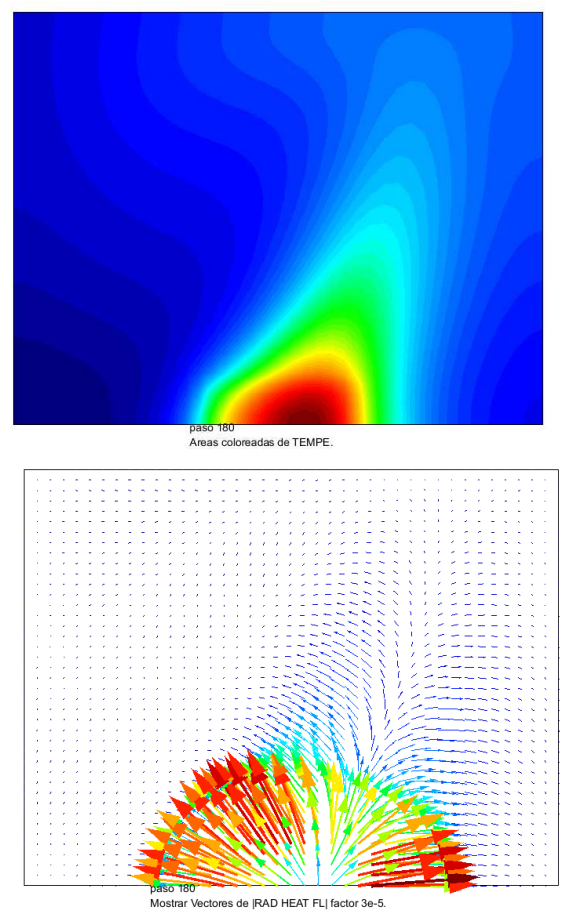

Matso ion

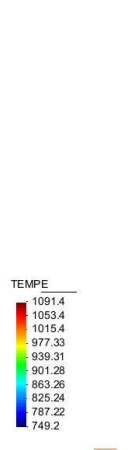

cill

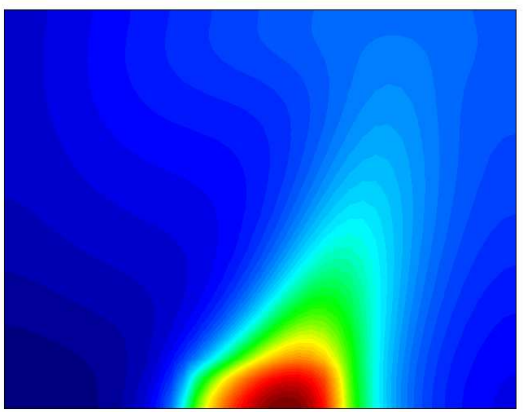

paso 180
Araas coloreadas de TEMPE.
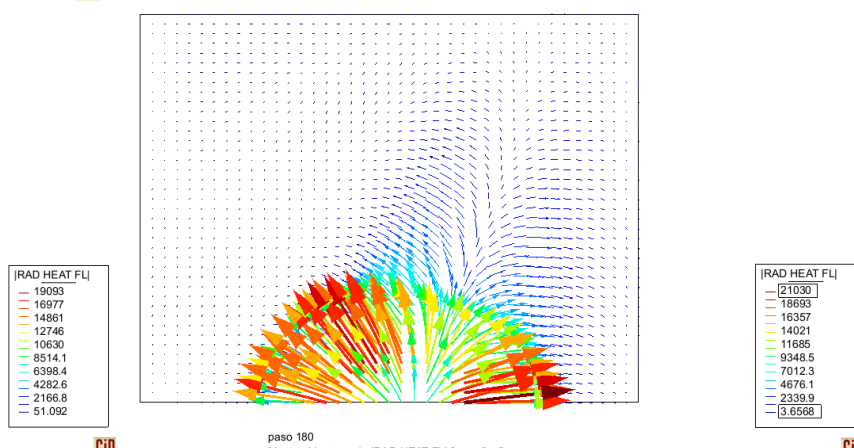

Figure 15: Temperature distribution and radiative heat flux distribution over plane $y=L / 2$ when splitting radiative term as $4 \kappa \sigma_{B}\left(T_{h}+\widetilde{T}\right)^{4}$ (left) and not splitting $4 \kappa \sigma_{B} T_{h}^{4}$ (right))

[7] B. Davison. Neutron Transport Theory. Oxford University Press, London, 1958.

[8] B. Dubroca, M. Seaïd, and I. Teleaga. A consistent approach for the coupling of radiation and hydrodynamics at low mach number. Journal of Computational Physics, 225:1039-1065, 2007.

[9] T.J.R. Hughes. Multiscale phenomena: Green's function, the Dirichlet-to-Neumann formulation, subgrid scale models, bubbles and the origins of stabilized formulations. Computer Methods in Applied Mechanics and Engineering, 127:387-401, 1995.

[10] T.J.R. Hughes, G. Emgel, L. Mazzei, and M.G. Larson. The continuous Galerkin method is locally conservative. Journal of Computational Physics, 163:467-488, 2000.

[11] S. Hun-Kang and T.-H. Song. Finite element formulation of the first- and second- order discrete ordinates equations for radiative heat transfer calculation in three dimensional participating media. Journal of Quantitative Spectroscopy \& Radiative Transfer, 109:2094-2107, 2008.

[12] K.D. Lathrop. Use of discrete-ordinate methods for solution of photon transport problems. Nuclear Science and Engineering, 24:381-388, 1966.

[13] M.F. Modest. Photon-gas formulation of the differential approximation in radiative transfer. Letters in Heat and Mass Transfer, 3:111-116, 1976.

[14] M.F. Modest. Radiative heat transfer. Academic Press, 2003.

[15] J. Truelove. Discrete-ordinate solution of the radiation transport equation. ASME Journal of Heat Transfer, 109:1048-1051, 1987. 

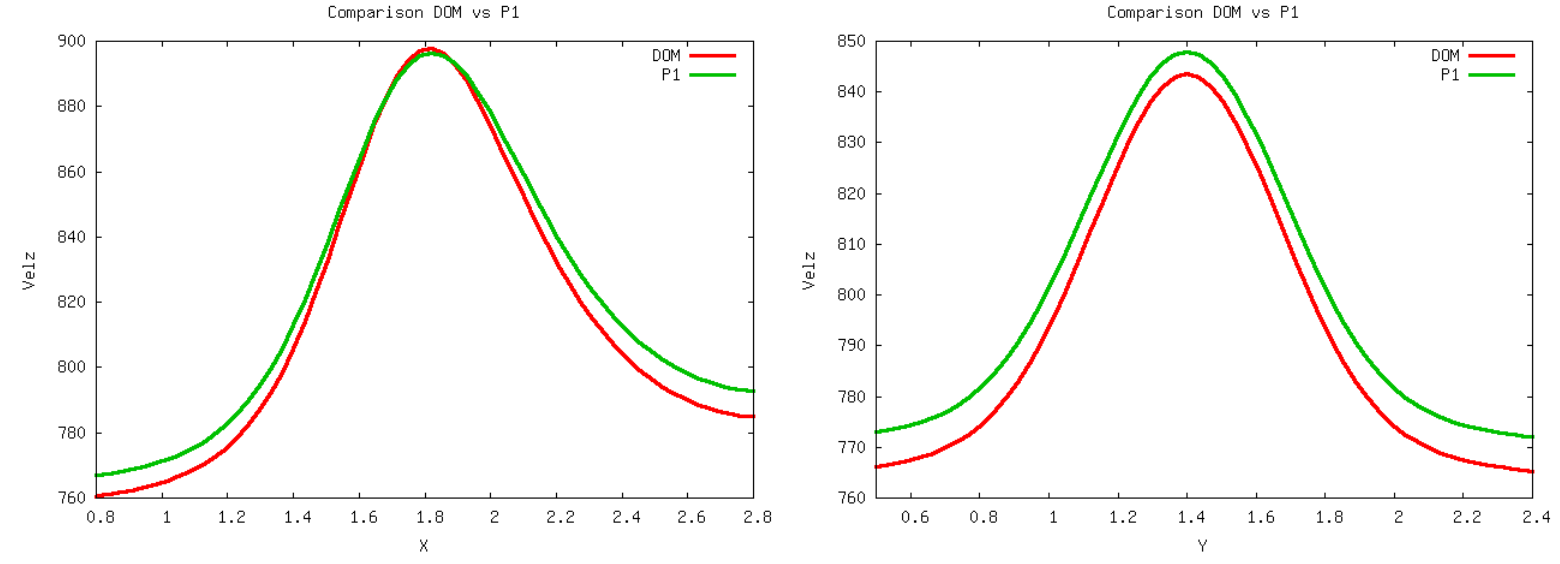

Figure 16: Temperature distribution along $x$ and $y$ direction using the P1 method and the DOM 